\title{
$\S 10$ Beweiserleichterungen bei der Haftung für den Missbrauch von Zugangsdaten im Internet
}

Neben der materiellen Rechtslage ist für den Geschäftsgegner entscheidend, ob er seinen Anspruch beweisen kann. Die grundsätzliche Regel bei der Beweislastverteilung im Zivilprozess ist, dass jede Partei, die sie begünstigenden Tatsachen zu beweisen hat ${ }^{1}$ Der Geschäftsgegner muss nach diesem Grundsatz beweisen, dass der Account-Inhaber selbst oder ein Dritter mit dessen Einverständnis eine elektronische Willenserklärung abgegeben hat. Dieser Beweis ist dem Geschäftsgegner durch den Augenscheinsbeweis mit dem elektronischen Dokument ( 371 Abs. 1 S. 2 ZPO) schwer bis gar nicht möglich. Die Echtheit der Erklärung kann der Geschäftsgegner nur in den seltensten Fällen im Rahmen eines Vollbeweises beweisen 2 Zum Beweis der Echtheit einer elektronischen Erklärung gehört sowohl die Authentizität, also die Urheberschaft der Erklärung, sowie deren Integrität, ein Ausschluss von nachträglichen Veränderungen 3 Durch umfassende Informationen zum verwendeten Rechner, der IP-Adresse und der Sicherheitsinfrastruktur des Authentisierungsnehmers wäre ein Vollbeweis möglich ${ }^{4}$ Diese Informationen sind jedoch häufig nur staatlichen Behörden zugänglich ${ }_{5}^{5}$ Die Echtheit der Erklärung lässt sich nur durch Begleitumstände, nicht durch die Erklärung selbst beweisen ${ }^{6}$ Dies hat zur Folge, dass ein elektronischer Vertragsschluss faktisch nicht mit einem Vollbeweis geführt werden kann 7

Diese Beweisschwierigkeiten sind insbesondere bei der Inanspruchnahme des Account-Inhabers relevant. Ohne eine Beweiserleichterung kann er sich durch Bestreiten vom Vertrag lösen, er hat quasi ein „Widerrufs-

1 Diese sog. Rosenbergsche Formel wurde entwickelt von Rosenberg $^{5}$, S. 98 ff. und ist nunmehr allgemein anerkannt $B G H$, Urteil v. 18. 5. 2005, VIII ZR 368/03 - NJW 2005, 2395, 2396; Adolphsen ${ }^{4}$, § 23 Rn. 61; Jauernig/Hess ${ }^{30}$, § 50 Rn. 11; Lüke ${ }^{10}$, Rn. 277; Paulus ${ }^{4}$, Rn. 416.

2 Borges, Verträge, S. 486; ders., Elektronischer Identitätsnachweis, S. 230; Borges/ Schwenk/Stuckenberg/Wegener, S. 302.

3 Bergfelder, S. $87 \mathrm{ff}$.

4 Borges, Verträge, S. 487.

5 Ebd. S. 487.

6 Ebd. S. 488.

7 LG Bonn, Urteil v. 19. 12. 2003, 2 O 472/03 - MMR 2004, 179, 180; Borges, NJW $2005,3313,3316$. 
recht kraft Beweislastverteilung 8 . Diese Problematik von Schutzbehauptungen kann über die freie richterliche Beweiswürdigung ( $\$ 286$ Abs. 1 S. 1 ZPO) oder über Beweiserleichterungen gelöst werden. Nachfolgend sollen zunächst Lösungen über mögliche Beweiserleichterung untersucht werden. Der Behauptung, der Haftung für den Missbrauch von Zugangsdaten sei kein materielles, sondern nur ein prozessuales Problem.9 $9^{9}$ kann nicht zugestimmt werden. Über eine prozessuale Lösung kann nur Schutzbehauptungen und Ausreden des sich vom Vertrag lösen wollenden Account-Inhaber begegnet werden. Materiell stellen sich auch bei wahrheitsgemäß aufgeklärtem Sachverhalt Fragen der Zurechnung. Wegen der Beweisschwierigkeiten des Geschäftsgegners ist zu erwägen, ob Beweiserleichterungen für den Vertragsschluss über Accounts im Internet in Betracht kommen. Dazu sollen zunächst unterschiedliche Formen der Beweiserleichterung betrachtet werden, um zu prüfen, ob deren Voraussetzungen beim Missbrauch von Zugangsdaten im Internet vorliegen.

\section{Formen der Beweiserleichterung}

774 Es existieren verschiedene Formen der Beweiserleichterung. Unter dem Begriff der Beweiserleichterung werden hier sämtliche Formen der Abweichung von der Grundregel, dass eine Partei sie begünstigende Tatsachen zu beweisen hat, verstanden 10 Dem engen Verständnis, dass nur Erleichterungen bei der Beweiswürdigung erfasst sind ${ }^{11}$ wird nicht gefolgt. Folgend werden Beweiserleichterungen in Form der Beweislastumkehr, des Anscheinsbeweises und der sekundären Darlegungslast untersucht.

\section{Beweislastumkehr mit und ohne tatsächlicher Vermutung}

775 Die stärkste Form der Beweiserleichterung ist die Umkehr der Beweislast, weil derjenige zu dessen Lasten die Beweislast umgekehrt wurde, den vol-

$8 \quad$ Mankowski, CR 2003, 44; ders., MMR 2004, 181.

9 Probandt, UFITA 98 (1984), 9, 18.

10 So auch $B G H$, Urteil v. 7. 6. 1988, VI ZR 91/87 (Limonadenflasche) - BGHZ 104, 323, 333; Urteil v. 27. 4. 2004, VI ZR 34/03 (Beckenringfraktur) - BGHZ 159, 48, 53.

11 Laumen, NJW 2002, 3739, 3743; Prütting, in: Baumgärtel ${ }^{2}$, § 19 Rn. 7. 
len Gegenbeweis erbringen muss. In der Rechtsprechung haben sich zwei Formen der Beweislastumkehr herausgebildet, die folgend vorgestellt werden sollen.

\section{a) Umkehr der Beweislast}

Eine Beweislastumkehr kommt in seltenen Fällen in Betracht, in denen methodisch eine Abweichung vom Gesetz begründbar ist ${ }^{12}$ Für die Umkehr der Beweislast haben sich in der Rechtsprechung verschiedene Fallgruppen herausgebildet ${ }^{13}$ die folgend kurz vorgestellt werden, um anschließend herauszufiltern, mit welcher Begründung eine Beweislastumkehr erfolgen kann.

Eine Fallgruppe der Beweislastumkehr ist die Produzentenhaftung ${ }^{14}$ Dabei erfolgt eine Beweiserleichterung durch die Abgrenzung der Gefahrenbereiche $\sqrt{15}$ Der Produzent könne seinen komplexen Herstellungsprozess mit verschachtelter Arbeitsteilung sowie verwickelten technischen, chemischen oder biologischen Vorgängen, im Gegensatz zu seinem Prozessgegner, überblicken ${ }^{16}$ Ferner sei die hinter $\S \S 831,836$ BGB stehende Wertung, dass dem Beherrscher einer Gefahrenquelle das Risiko der Unaufklärbarkeit anzulasten ist, auf die Produzentenhaftung übertragbar ${ }^{17}$ Auch für kleine Betriebe mit einem überschaubaren Herstellungsprozess sei die Beweislastumkehr anwendbar, weil der Herstellungsvorgang in die Organisationssphäre des Herstellers fällt, in die der Prozessgegner keinen Einblick hat ${ }^{18}$ Ferner müsse der Hersteller eines Produkts dessen Fehlerfreiheit durch Ausgangskontrollen überprüfen, weil ansonsten die Beweisführung für den Zeitpunkt

12 Prütting, in: Baumgärtel $^{2}, \S 19$ Rn. 8.

13 Dazu Musielak, Grundlagen, S. $132 \mathrm{ff}$.

14 Dazu Jauernig/Hess ${ }^{30}, \S 50$ Rn. 34; Prütting, in: Baumgärtel ${ }^{2}$, § 19 Rn. 13; Riehm, JZ 2006, 1035, 1042 ff.; Rosenberg/K. H. Schwab/Gottwald ${ }^{17}$, 115 Rn. 28 f.

15 Prütting, in: Baumgärtel $^{2}, \S 19$ Rn. 16.

$16 B G H$, Urteil v. 26. 11. 1968, VI ZR 212/66 (Hühnerpest) - BGHZ 51, 91, 105.

$17 B G H$, Urteil v. 26. 11. 1968, VI ZR 212/66 (Hühnerpest) - BGHZ 51, 91, 106; Urteil v. 24. 11. 1976, VIII ZR 137/75 (Schwimmschalter) - BGHZ 67, 369, 362; Urteil v. 19. 11. 1991, VI ZR 171/91 (Hochzeitsessen) - BGHZ 116, 104, 108.

$18 B G H$, Urteil v. 19. 11. 1991, VI ZR 171/91 (Hochzeitsessen) - BGHZ 116, 104, 109; noch offen gelassen im Urteil v. 26. 11. 1968, VI ZR 212/66 (Hühnerpest) BGHZ 51, 91, 107. 
der Mangelhaftigkeit des Produkts schwer fällt ${ }^{19}$ Unterlässt er Ausgangskontrollen finde ebenfalls eine Beweislastumkehr statt 20 Mit dieser Beweislastumkehr bei der Produkthaftung besteht ökonomisch betrachtet eine Lage, die einer Gefährdungshaftung für diese Produkte sehr nahe kommt ${ }^{21}$ Insofern erklärt sich die teleologische Begründung, dass der Hersteller diese Gefahren besser versichern könne als der Verwender des Produkts 22

Die zweite Fallgruppe betrifft eine Umkehr der Beweislast für den Kausalitätsbeweis bei der Arzthaftung ${ }^{23}$ Diese Beweislastumkehr für den Kausalitätsbeweis komme in Betracht, wenn der Arzt einen groben Behandlungsfehler begangen hat ${ }^{24}$ Zwar kann der Arzt die Kausalität nur ebenso schwer beweisen wie der Patient ${ }^{25}$ Durch einen groben Fehler schaffe der Arzt jedoch eine Lage, in der nicht mehr erkennbar ist, was den Fehler verursacht hat, was ihm zu Lasten fällt ${ }^{26}$ Selbst beim Vorliegen eines groben Behandlungsfehlers sei die Beweislastumkehr jedoch ausnahmsweise ausgeschlossen, wenn der Ursachenzusammenhang äußerst unwahrscheinlich ist ${ }^{27}$ Ebenso scheidet bei fehlendem Risikozusammenhang die Beweislastumkehr aus ${ }^{28}$ Das Vorliegen dieser Ausnahmen hat jedoch der Arzt zu beweisen ${ }^{29}$ Eine Beweislastumkehr bei der Arzthaftung komme ebenfalls in Betracht, wenn der Arzt seine Dokumentationspflicht verletzt ${ }^{30}$ Der Patient leide bei mangelnder Dokumentation an einer Beweisnot bezüglich möglicher Behandlungsfehler, sodass ihm durch eine Beweislastumkehr der schwierige Beweis erleichtert wird 31

$19 B G$, Urteil v. 7. 6. 1988, VI ZR 91/87 (Limonadenflasche) - BGHZ 104, 323, 334.

20 Ebd. 334.

21 Schäfer/C. Ott ${ }^{5}$, S. 224. Ohne die rechtsökonomischen Erwägungen auch Prütting, in: Baumgärtel ${ }^{2}, \S 19$ Rn. 21.

$22 B G H$, Urteil v. 26. 11. 1968, VI ZR 212/66 (Hühnerpest) - BGHZ 51, 91, 104 f.; Prütting, in: Baumgärtel ${ }^{2}, \S 19$ Rn. 18.

23 Dazu Jauernig/Hess ${ }^{30}$, § 50 Rn. 38; Musielak, in: FG 50 Jahre BGH, Bd. 3, 193, 217; Prütting, in: Baumgärtel ${ }^{2}$, § 19 Rn. 25; Rosenberg/K. H. Schwab/Gottwald ${ }^{17}$, $\S 115$ Rn. 24 ff.

$24 B G H$, Urteil v. 27. 4. 2004, VI ZR 34/03 (Beckenringfraktur) - BGHZ 159, 48, 55; Urteil v. 12. 2. 2008, VI ZR 221/06 - NJW 2008, 1381, Rn. 13.

$25 B G H$, Urteil v. 19. 11. 1955, VI ZR 214/54 - VersR 1956, 499, 500.

26 Ebd. 500.

$27 B G H$, Urteil v. 27. 4. 2004, VI ZR 34/03 (Beckenringfraktur) - BGHZ 159, 48, 55.

28 Ebd. 55.

29 Ebd. 55.

30 Prütting, in: Baumgärtel ${ }^{2}, \S 19$ Rn. 32.

$31 B G H$, Urteil v. 27.6. 1978, VI ZR 183/76 - BGHZ 72, 132, 136 ff. 
Die dritte Fallgruppe ist die Beweislastumkehr bei Beweisvereitelung 32 Zerstört oder entzieht jemand einem aktuellen oder potentiellen Prozessgegner schuldhaft Beweise, tritt eine Beweislastumkehr zu Lasten desjenigen ein, der die Beweise zerstört oder entzogen hat ${ }^{33}$ Diese Beweislastumkehr bei der Beweisverteilung lasse sich aus dem Rechtsgedanken der $\S \S 427$, 441 Abs. 3 S. 3, 444, 446, 453 Abs. 2, 454 Abs. 1 ZPO ableiten ${ }^{34}$ Das schuldhafte Beeinträchtigen der Beweislage des Gegners in einem aktuellen oder künftigen Prozess rechtfertige es, den Beweisvereiteler die Nachteile der entstehenden Beweisnot tragen zu lassen ${ }^{35}$ Der daraus entstehende Schuldvorwurf muss sich dabei sowohl auf die Zerstörung oder Entziehung des Beweisobjekts als auch auf die Benachteiligung des Gegners in einem Prozess beziehen 36

Als Gemeinsamkeit der drei Fallgruppen lässt sich herausfiltern, dass die Partei, zu Gunsten derer die Beweislastumkehr in Ausnahmefällen angenommen wird, unter der schweren Beweisnot leidet, dass sie gewisse Tatsachen nicht beweisen kann. Bei der Produzentenhaftung entsteht die Beweisnot dadurch, dass sich der Vorgang komplett in der Sphäre des Herstellers abspielt, in die die Gegenpartei keinen Einblick hat, und der Hersteller diese Vorgänge jedoch gestalten und überblicken kann. Bei der Beweisvereitelung entsteht die Beweisnot durch ein vorwerfbares Verhalten der Gegenseite. Der Vernichter von Beweisen solle aus Billigkeitserwägungen keine Vorteile durch sein schuldhaftes Handeln erhalten. Die Begründung bei der Arzthaftung ähnelt jener der Beweisverteilung, weil entscheidend ist, dass der Arzt durch seinen groben Behandlungsfehler den Beweis des Patienten erschwert. Aus den drei Fallgruppen ist noch eine unausgesprochene Voraussetzung abzuleiten. Dem Begünstigten darf es nicht möglich sein, die Beweise selbst herzustellen. Ansonsten könnte ihm die Beweisnot ebenso vorgeworfen werden wie der anderen Partei. Zusammenfassend lässt sich festhalten, dass eine Beweislastumkehr in Betracht kommt, wenn der Begünstigte an einer Beweisnot dadurch leidet, dass der Prozessgegner diese

32 Dazu Jauernig/Hess ${ }^{30}$, § 50 Rn. 30; Leipold, in: Stein/Jonas ${ }^{22}$, § 286 ZPO Rn. 187 ff.; Prütting, in: Baumgärtel ${ }^{2}$, § 19 Rn. 36; Rosenberg/K. H. Schwab/ Gottwald $^{17}$, § 115 Rn. 19 ff.; Schilken, Zivilprozessrecht ${ }^{6}$, Rn. 507.

$33 B G H$, Urteil v. 23. 9. 2003, XI ZR 380/00 - NJW 2004, 222; Urteil v. 23. 11. 2005, VIII ZR 43/05 - NJW 2006, 434, Rn. 23.

$34 B G H$, Urteil v. 23. 11. 2005, VIII ZR 43/05 - NJW 2006, 434, Rn. 23.

$35 B G H$, Urteil v. 23. 11.2005, VIII ZR 43/05 - NJW 2006, 434, Rn. 23; Musielak, Grundlagen, S. 136.

BGH, Urteil v. 23. 9. 2003, XI ZR 380/00 - NJW 2004, 222. 
schuldhaft hervorgerufen hat oder dass sich die Vorgänge in der Sphäre des Prozessgegners ohne Einblicksmöglichkeit des Begünstigen abspielen und der Begünstigte die Beweisnot nicht anderweitig verhindern kann.

\section{b) Tatsächliche Vermutung}

781 Ferner lassen sich in der Rechtsprechung Fälle finden, bei denen eine Beweislastumkehr als Folge einer tatsächlichen Vermutung angenommen wird. Beispielsweise besteht eine tatsächliche Vermutung, dass eine Privaturkunde vollständig is ${ }^{37}$ oder dass die GEMA zur Wahrnehmung der Rechte an öffentlich aufgeführten Musiktiteln berechtigt ist ${ }^{38}$ Tatsächliche Vermutungen sind von gesetzlichen Vermutungen ( 292 S. 1 ZPO) ${ }^{39}$ zu unterscheiden. Im Gegensatz zu diesen sind jene nicht von $\S 292$ ZPO erfasst ${ }^{40}$ Der Begriff der tatsächlichen Vermutung wird unterschiedlich verwendet ${ }^{41}$ Zum einen kann eine tatsächliche Vermutung als Grundlage eines Anscheinsbeweises verstanden werden 42 Zum anderen wird eine tatsächliche Vermutung zur Begründung einer sekundären Darlegungslast verwen$\operatorname{det}{ }^{43}$ Ferner kann als tatsächliche Vermutung die Umkehr der Beweislast verstanden werden ${ }^{44}$ Letzterem Verständnis der tatsächlichen Vermutung wird vorgeworfen, dass eine Erwägung mit Erfahrungswissen ausschließlich im Bereich der Beweiswürdigung und nicht der Beweislast anzusiedeln sei ${ }^{45}$ Ein Umkehren der Beweislast mit Hilfe der tatsächlichen Vermutung

$37 B G H$, Urteil v. 11. 11. 1977, V ZR 105/75 - WM 1978, 244.

$38 B G H$, Urteil v. 5.6. 1985, I ZR 53/83 (GEMA-Vermutung I) - BGHZ 95, 274, $276 \mathrm{f}$.

39 Dazu Lüke ${ }^{10}$, Rn. 266; Paulus ${ }^{4}$, Rn. 427; Musielak, Grundkurs ${ }^{11}$, Rn. 480.

40 Lüke ${ }^{10}$, Rn. 266; Musielak, JA 2010, 561; Prütting, in: MüKo-ZPO ${ }^{4}$, 292 Rn. 7; a.A. Bruns ${ }^{2}$, Rn. 171c.

41 Allner, S. 21 ff.; Prütting, Gegenwartsprobleme, S. 57.

42 So BGH, Urteil v. 9. 10. 2009, V ZR 178/08 - NJW 2010, 363, Rn. 15; Schellham$m^{14}{ }^{14}$, Rn. 519; Baumgärtel, in: FS Schwab, 43, 50.

43 BGH, Urteil v. 12.5.2010, I ZR 121/08 (Sommer unseres Lebens) - BGHZ 185, 322, Rn. 12; Urteil v. 15. 11.2012, I ZR 74/12 (Morpheus) - NJW 2013, 1441, Rn. $33 \mathrm{f}$.

44 So $B G H$, Urteil v. 11. 11. 1977, V ZR 105/75 - WM 1978, 244; Urteil v. 5. 6. 1985, I ZR 53/83 (GEMA-Vermutung I) - BGHZ 95, 274, 276.

45 Allner, S. 55 ff.; Laumen, in: Prütting/Gehrlein ${ }^{5}$, § 292 ZPO Rn. 7; Prütting, Gegenwartsprobleme, S. 55; Rosenberg/K. H. Schwab/Gottwald ${ }^{17}$, § 113 Rn. 37. 
umgehe häufig die Voraussetzungen der Rechtsfortbildung ${ }^{46}$ Unabhängig davon, wie berechtigt diese Kritik ist, wird die tatsächliche Vermutung im Folgenden im Bereich der Beweislast angesiedelt. Vielfach lässt sich in der Rechtsprechung eine tatsächliche Vermutung mit der Folge einer Beweislastumkehr finden. Für diese wird hier der Begriff der tatsächlichen Vermutung verwendet. Dadurch wird eine begriffliche Trennung zwischen dem Problem der Beweiswürdigung mit dem Anscheinsbeweis und der Beweislastverteilung durch die tatsächliche Vermutung ermöglicht.

Eine tatsächliche Vermutung mit der Folge der Umkehr der Beweislast wird von der Rechtsprechung häufig ohne nähere Begründung angenommen. Dadurch kann die dogmatische Herleitung der tatsächlichen Vermutung ebenso wie deren Voraussetzungen nur schwer bestimmt werden. Beispielsweise wird ohne nähere Begründung bei Wettbewerbsverstößen die Wiederholungsgefahr tatsächlich vermutet und kann insbesondere durch eine strafbewehrte Unterlassungserklärung widerlegt werden ${ }^{47}$ Eine inhaltsgleiche tatsächliche Vermutung besteht auch bei der Wiederholungsgefahr bezüglich der Verwendung unwirksamer AGB ${ }^{48}$ Bei 20-jähriger Durchführung einer vom OHG-Gesellschaftsvertrag abweichenden Gewinnverteilung spreche eine tatsächliche Vermutung für die verbindliche Abänderung unter Verzicht auf die Schriftform ${ }^{49}$ Bei Verstoß gegen eine DIN-Norm besteht eine tatsächliche Vermutung dafür, dass Risiken, die die Norm verhindern soll, kausal auf der Verletzung der Norm beruhen 50 Erschwerend kommt bei der Bestimmung der Voraussetzungen hinzu, dass der Begriff, der tatsächlichen Vermutung nicht einheitlich verwendet wird. Teilweise wird eine tatsächliche Vermutung im Bereich der Produkthaftung angenommen ${ }^{51} \mathrm{ob}-$ wohl dort bereits aus anderem Grund eine Beweislastumkehr angenommen wird.

Einige Entscheidungen geben jedoch Einblick in die Erwägungsgründe, unter welchen Voraussetzungen eine tatsächliche Vermutung anzunehmen ist. Beispielsweise die tatsächliche Vermutung, dass eine Privaturkunde

46 Laumen, in: Baumgärtel $^{2}$, § 14 Rn. 21; Prütting, Gegenwartsprobleme, S. 54 f.; ders., in: MüKo-ZPO ${ }^{4}$ \$ 292 Rn. 28.

$47 B G H$, Urteil v. 8. 2. 1980, I ZR 22/78 (Grand Prix) - NJW 1980, 1793, 1794.

$48 B G H$, Urteil v. 9.7. 1981, VII ZR 123/80 - BGHZ 81, 222, 225 f.

$49 B G H$, Urteil v. 17. 1. 1966, II ZR 8/64 - NJW 1966, 826, 827.

$50 B G$, Urteil v. 19.4. 1991, V ZR 349/89 - BGHZ 114, 273, 276, wobei jedoch nur von einer ,widerleglichen Vermutung“ gesprochen wird.

51 So BGH, Urteil v. 12. 11. 1991, VI ZR 7/91 (Kindertee) - BGHZ 116, 60, 73. 
vollständig ist 52 wurde so begründet, dass sich daraus Voraussetzungen für deren Annahme herausarbeiten lassen. Eine tatsächliche Vermutung beruht auf allgemeinen Erfahrungssätzen aus der Lebenserfahrung 53 Häufig wird die tatsächliche Vermutung über Wahrscheinlichkeitserwägungen begründet ${ }^{54}$ Der Behauptung, dass eine überwiegende Wahrscheinlichkeit bereits zur Annahme einer tatsächlichen Vermutung ausreiche ${ }^{55}$ kann nicht zugestimmt werden ${ }^{56}$ Bei der GEMA-Vermutung beruht die überwiegende Wahrscheinlichkeit an der Quasi-Monopolstellung der GEMA bei der Wahrnehmung von Urheberrechten an gewissen Werken, sodass mit hoher Wahrscheinlichkeit bei der Verwendung von Musik von der GEMA wahrgenommene Rechte betroffen sind ${ }^{57}$ Ferner solle der gesetzgeberische Wille leistungsfähige Verwertungsgesellschaften zu schaffen, mit der Beweiserleichterung verwirklicht werden 58 Als Voraussetzung lässt sich somit herausfiltern, dass aufgrund der Lebenserfahrung mit hoher Wahrscheinlichkeit auf eine Tatsache geschlossen werden kann. Die effektive Rechtsdurchsetzung wegen ansonsten bestehender Beweisnot kann ebenfalls zur Begründung herangezogen werden.

784 Die tatsächliche Vermutung führt objektiv zu einer Umkehr der Beweislast für das Vorliegen der vermuteten Haupttatsachen 59 Um die tatsächliche Vermutung zu entkräften, muss der volle Gegenbeweis erbracht werden 60 Eine solche Beweispflicht kommt daher nur in Betracht, wenn eine Tatsache außerhalb des von der darlegungspflichtigen Partei zu klärendem Geschehensablaufs stattfindet, während der Prozessgegner Kenntnis habe und auch Aufklärung von ihm verlangt werden kann 61 Die tatsächliche Vermutung

$52 B G H$, Urteil v. 11. 11. 1977, V ZR 105/75 - WM 1978, 244; Urteil v. 5. 7. 2002, V ZR 143/01 - NJW 2002, 3164, 3165. Dazu Allner, S. 42 ff.

53 Allner, S. 37, 65; Huber, in: Musielak ${ }^{10}$, § 292 ZPO Rn. 1; Lüke ${ }^{10}$, Rn. 266; Musielak, Grundkurs ${ }^{11}$, Rn. 480.

54 Wassermeyer, S. 35 m.w.N.

55 Bruns $^{2}, \mathrm{Rn} .171 \mathrm{c}$.

56 Laumen, in: Baumgärtel ${ }^{2}, \S 14$ Rn. 7.

$57 B G H$, Urteil v. 5. 6. 1985, I ZR 53/83 (GEMA-Vermutung I) - BGHZ 95, 274, 277; Urteil v. 13. 6. 1985, I ZR 35/83 (GEMA-Vermutung II) - BGHZ 95, 285, 290. $B G H$, Urteil v. 13.6. 1985, I ZR 35/83 (GEMA-Vermutung II) - BGHZ 95, 285, $290 \mathrm{f}$.

59 Förschler/Steinle ${ }^{7}$, Rn. 817; Laumen, in: Prütting/Gehrlein ${ }^{5}$, § 292 ZPO Rn. 6; Musielak, JA 2010, 561.

$60 B G H$, Urteil v. 11.11.1977, V ZR 105/75 - WM 1978, 244.

61 Musielak, Grundkurs ${ }^{11}$, Rn. 403; Prütting, in: MüKo-ZPO ${ }^{4}$ § 286 Rn. 131. 
muss dem Beweis des Gegenteils zugänglich sein ${ }^{62}$ Wenn der Gegner nicht die Möglichkeit hat, das Gegenteil zu beweisen, kommt eine tatsächliche Vermutung somit nicht in Betracht. Alternative Möglichkeiten schließen die Vermutung nicht aus, wie beispielsweise das häufige Falschbeurkunden eines Grundstückkaufvertrags ${ }^{63}$ Sie sind der Gegenstand eines möglichen Gegenbeweises. Im Gegensatz zur gesetzlichen Vermutung muss bei der tatsächlichen Vermutung nicht nur die Vermutungsbasis vorgetragen werden, sondern auch die vermutete Tatsache 64

\section{Anscheinsbeweis}

Der Anscheinsbeweis, auch Beweis des ersten Anscheins oder prima-facieBeweis genannt ${ }^{65}$ ist eine Form der mittelbaren Beweisführung im Rahmen der freien Beweiswürdigung (vgl. § 286 Abs. 1 S. 1 ZPO) durch den Richter im Zivilprozess ${ }^{66}$ Beim Anscheinsbeweis wird von einem typischen $\mathrm{Ge}$ schehensverlauf nach der allgemeinen Lebenserfahrung auf das Vorliegen einer bestimmten Ursache oder Folge geschlossen 67 Der Anscheinsbeweis ist nicht gesetzlich normiert, wird aber in $\S 371 \mathrm{a}$ ZPO vorausgesetzt ${ }^{68} \mathrm{Er}$ statuiert keine Beweislastumkehr, sondern verhindert eine non-liquet-Situation 69 Für die Begründung eines Anscheinsbeweises wird häufig der Gedanke der Sphären herangezogen, dass jede Partei für ihren Gefahrenbereich verantwortlich ist und Nachteile zu tragen hat, die durch die Unaufklärbarkeit der aus ihrer Sphäre stammenden Tatsachen entstehen ${ }^{70}$ Dabei erfüllt der Anscheinsbeweis häufig die Funktion eines Irgendwie-Beweises 71 Kann beispielsweise das Verschulden nicht voll bewiesen werden, führen jedoch die meisten und die wahrscheinlichen Möglichkeiten zum gleichen rechtlichen Ergebnis, wird angenommen, dass ein Verschulden, egal welcher Art, vorliegt.

$62 B G H$, Urteil v. 5. 7. 2002, V ZR 143/01 - NJW 2002, 3164, 3165.

$63 B G H$, Urteil v. 11. 11. 1977, V ZR 105/75 - WM 1978, 244.

$64 B G H$, Urteil v. 9. 10. 2009, V ZR 178/08 - NJW 2010, 363, Rn. 13.

65 Adolphsen ${ }^{4}, \S 23$ Rn. 38; Musielak, Grundlagen, S. 83.

66 Prütting, in: MüKo-ZPO ${ }^{4}$ \& 286 Rn. 48; Schellhammer ${ }^{14}$, Rn. 518.

67 Lüke $^{10}$, Rn. 279; Foerste, in: Musielak ${ }^{10}$, § 286 ZPO Rn. 23; Paulus ${ }^{4}$, Rn. 424.

68 Jauernig/Hess ${ }^{30}$, § 50 Rn. 11; Musielak, Grundkurs ${ }^{11}$, Rn. 468.

69 Adolphsen $^{4}$, § 23 Rn. 40.

70 Hanau, Handeln unter fremder Nummer, S. 53.

71 Prütting, Gegenwartsprobleme, S. 110; Kollhosser, AcP 165 (1965), 46, 62 f. 
786 Ein Erfahrungssatz, der einen Anscheinsbeweis begründet, basiert auf der Beobachtung, dass beim Eintreten einer bestimmten Tatsache nicht zwingend, aber mit hoher Wahrscheinlichkeit oder aufgrund eines Musters auf das Vorliegen einer Ursache oder Folge zu schließen ist. ${ }^{72}$ Er muss so stark sein, dass er die volle Überzeugung des Gerichts von der behaupteten Tatsache begründet ${ }^{73}$ Typisch ist ein Geschehensablauf, wenn er nach der Erfahrung des täglichen Lebens durch das Regelmäßige, Übliche, Gewöhnliche und Häufige seines Ablaufs sein Gepräge erhältt ${ }^{74}$ Ein solcher Erfahrungssatz entsteht durch die induktive Verallgemeinerung ${ }^{75}$ Dieser Erfahrungssatz ist zu unterscheiden von einem einfachen Erfahrungssatz, der sich dadurch auszeichnet, dass die Wahrscheinlichkeit, wenn die eine Tatsache vorliegt, auch die Ursache oder Folge vorliegt, nicht ausreichend hoch ist, um für die volle richterliche Überzeugung auszureichen 76

787 Für die Begründung eines Erfahrungssatzes für einen Anscheinsbeweis kommt es nicht auf eine empirische Statistik an 77 Bei der Wahrscheinlichkeit handelt es sich nämlich um eine Aussage bezüglich einer statistischen Masse, wohingegen im Einzelfall die Aussage entweder zutrifft oder nicht zutrifft 78 Vielmehr kommt es darauf an, dass nach logischen Erwägungen, die von der allgemeinen Lebenserfahrung gedeckt sind, eine hohe Wahrscheinlichkeit für den typischen Geschehensablauf spricht 79 Es reiche nicht aus, dass bei zwei verschiedenen Möglichkeiten eine wahrscheinlicher ist als die andere ${ }^{80}$ Diese Anforderung wird jedoch in der Rechtsprechung nicht konsequent durchgehalten. Ein Anscheinsbeweis kommt nicht in Betracht, wenn der Begünstigte seine Beweisnot anderweitig verhindern kann. Bei einem versendeten Brief steht dem Absender beispielsweise durch die

72 Laumen, in: Baumgärtel ${ }^{2}$, § 12 Rn. 12; Musielak, Grundlagen, S. 94 ff.; Prütting, in: MüKo-ZPO ${ }^{4}$ \& 286 Rn. 58.

73 Laumen, in: Prütting/Gehrlein ${ }^{5}$, § 286 ZPO Rn. 26; Leipold, in: Stein/Jonas ${ }^{22}$, $\S 286$ ZPO Rn. 130.

$74 \quad B G H$, Urteil v. 27. 5. 1957, II ZR 132/56 (Einschreibbrief) - BGHZ 24, 308, 312; Urteil v. 18. 3. 1987, IVa ZR 205/85 - BGHZ 100, 214, 216.

75 Jungmann, ZZP 120 (2007), 459, 461; ders., in: Jahrbuch Junger Zivilrechtswissenschaftlicher 2007, 329, 344.

76 Prütting, Gegenwartsprobleme, S. 108; ders., in: MüKo-ZPO ${ }^{4}$ \& 286 Rn. 60.

$77 B G H$, Urteil v. 27.5. 1957, II ZR 132/56 (Einschreibbrief) - BGHZ 24, 308, 312; Prütting, Gegenwartsprobleme, S. 94.

78 Musielak, in: FG 50 Jahre BGH, Bd. 3, 193, $204 \mathrm{f.}$

79 Prütting, Gegenwartsprobleme, S. 106.

$80 B G H$, Urteil v. 27. 5. 1957, II ZR 132/56 (Einschreibbrief) - BGHZ 24, 308, 313. 
förmliche Zustellung oder ein Einschreiben mit Rückschein die Möglichkeit zu, einen sicheren Beweis zu schaffen ${ }^{81}$ Daher besteht insoweit kein Bedürfnis ihm mit einer Beweiserleichterung zu helfen.

Andererseits lassen sich auch Fälle feststellen, bei denen unter zwei oder mehr vergleichbar wahrscheinlichen Möglichkeiten, die leicht überwiegend wahrscheinliche gewählt wurde. Dies geschieht dort, wo Beweisnot herrscht und wo Erfahrungssätze, die sich durch Häufigkeit, Üblichkeit und Gleichmäßigkeit auszeichnen, fehlen. Dabei werden im Ausschlussverfahren unwahrscheinliche Geschehensabläufe eliminiert ${ }^{82}$ Dieser „Anscheinsbeweis ohne ersten Anschein ' 83 lässt sich in diversen Entscheidungen des $B G H$ wiederfinden ${ }^{84}$ Beim lautlosen Ertrinken eines Nichtschwimmers spreche beispielsweise ein Anscheinsbeweis für die Ursächlichkeit einer fehlenden Absperrung, wobei körperliche Mängel als Alternativmöglichkeit unwahrscheinlich sind, aber den Anscheinsbeweis erschüttern könnten 85 Bei der nicht vollbewiesenen Erkrankung mit Lues spreche ein Anscheinsbeweis dafür, dass gewisse Symptome für eine Lues und keine andere Krankheit sprechen und dass die Infektion auf eine fünf Jahre zurückliegende Bluttransfusion zuzuführen sei 86 Dabei reiche es aus, dass der vermeintlich typische Geschehensablauf in „Gutachten der Sachverständigen nicht ausgeschlossen“ wurde und dass zu der Frage wenig Erfahrungsmaterial vorliegt ${ }^{87}$ Ebenso spreche ein Anscheinsbeweis dafür, dass eine Ehefrau ihren Mann bei einer Bluttransfusion mit Lues angesteckt hat, obwohl nicht feststand, dass die Frau sich vor der Transfusion mit Lues angesteckt hatte 88 Ferner spreche ein Anscheinsbeweis dafür, dass sich der Unfall eines Gaststättenbesuchers beim Verlassen der Gaststätte und nicht bei einer späteren Rückkehr ereignete ${ }^{89}$ Diese Fälle haben gemeinsam, dass zahlreiche ver-

81 BGH, Urteil v. 27. 5. 1957, II ZR 132/56 (Einschreibbrief) - BGHZ 24, 308, 313; Prütting, Gegenwartsprobleme, S. 105.

82 Jungmann, ZZP 120 (2007), 459, 465; ders., in: Jahrbuch Junger Zivilrechtswissenschaftlicher 2007, 329, 346.

83 Jungmann, ZZP 120 (2007), 459.

84 Zu den Fällen Jungmann, ZZP 120 (2007), 459, 462 ff.; Kollhosser, AcP 165 (1965), 46, 75; Musielak, Grundlagen, S. $99 \mathrm{ff}$.

$B G H$, Urteil v. 3. 2. 1954, VI ZR 332/52 (Nichtschwimmer) - NJW 1954, 1119 , $1119 \mathrm{f}$.

$86 B G H$, Urteil v. 14. 12. 1953, III ZR 183/52 (Lues I) - BGHZ 11, 227, $230 \mathrm{f}$.

87 Ebd. 231.

$88 B G H$, Urteil v. 12. 2. 1957, VI ZR 303/56 (Lues II) - VersR 1957, 252.

$89 B G H$, Urteil v. 17. 12. 1953, III ZR 136/52 - VersR 1954, 401, 402. 
schiedene Möglichkeiten des Geschehensablaufs in Betracht kommen und sich keine überwiegend wahrscheinliche Möglichkeit herausbildet. Wahrscheinlich aus der einhergehenden Beweisnot wurde dennoch zu Gunsten des Geschädigten ein Anscheinsbeweis angenommen. Problem ist, dass wenn eine Tatsache für beide Seiten schwer zu beweisen ist, das Problem des Beweises und somit auch das Risiko, die Tatsache nicht beweisen zu können, auf die Gegenpartei verlagert wird ${ }^{90}$ Deswegen muss bei dem Anscheinsbeweis ohne ersten Anschein die sich auf den Anscheinsbeweis berufende Partei die Unwahrscheinlichkeit der auszuschließenden anderen Möglichkeiten beweisen 91

789 Die Anwendung des Anscheinsbeweises ist durch das Revisionsgericht überprüfbar 22 wodurch der Anscheinsbeweis die freie Beweiswürdigung unter die Kontrolle des Revisionsgerichts in typisierbaren Fällen stellt 93 Der Grund für die Bindung des Revisionsgerichts an die Tatsachenfeststellungen ist die Sachnähe bei der unmittelbaren Beweisaufnahme ${ }^{94}$ Bei typischen Sachverhalten hingegen ist keines der Gerichte sachnäher und es besteht ein Bedürfnis, typische Erfahrungssätze ebenso wie Rechtssätze in der Rechtsprechung zu vereinheitlichen 95

790 Für die Entkräftung des Anscheinsbeweises mittels eines Gegenbeweises, muss die Gegenseite den Anscheinsbeweis erschüttern ${ }^{96}$ indem sie konkrete Tatsachen behauptet, die in der Überzeugung des Gerichtes einen vom Erfahrungssatz abweichenden Geschehensablauf möglich erscheinen lässt ${ }^{97}$ Eine Beweislastumkehr, die den Beweis des Gegenteils zur Folge hätte, statuiert der Anscheinsbeweis gerade nicht ${ }^{98}$ Erhebliche Zweifel am Beweis können durch die ernsthafte Möglichkeit eines atypischen Gesche-

90 Prütting, Gegenwartsprobleme, S. 211.

91 Jungmann, ZZP 120 (2007), 459, 471; a.A. BGH, Urteil v. 14. 12. 1953, III ZR 183/52 (Lues I) - BGHZ 11, 227, 231.

$92 B G H$, Urteil v. 4. 10. 1983, VI ZR 98/82 - NJW 1984, 432, 433; Prütting, in: MüKo-ZPO 4 , 286 Rn. 66; Riehm, Abwägungsentscheidungen, S. 186; Laumen, in: Baumgärtel ${ }^{2}$, § 12 Rn. 41.

93 Riehm, Abwägungsentscheidungen, S. 91.

94 Ebd. S. 216.

95 Ebd. S. 216; a.A. wohl Kollhosser, AcP 165 (1965), 46, 55.

96 Adolphsen ${ }^{4}, \S 23$ Rn. 42; Jauernig/Hess ${ }^{30}$, § 50 Rn. 25.

97 Laumen, in: Baumgärtel ${ }^{2}$, § 12 Rn. 34; Schellhammer ${ }^{14}$, Rn. 519; Prütting, in: MüKo-ZPO ${ }^{4}$ \& 286 Rn. 65.

98 BGH, Urteil v. 5. 2. 1987, I ZR 210/84 (Raubpressungen) - BGHZ 100, 31, 34. 
hensablaufs im konkreten Fall begründet werden 99 Dazu müssen konkrete Tatsachen vorgetragen werden 100

Anscheinsbeweis und tatsächliche Vermutung erleichtern somit die Beweisführung für die beweisbelastete Partei. Der Unterschied zwischen den beiden ist, dass zum Widerlegen des Anscheinsbeweises das Erschüttern der Vermutungsgrundlage ausreicht, wohingegen bei der tatsächlichen Vermutung der volle Gegenbeweis erbracht werden muss. Die Voraussetzungen für das Anerkennen dieser beiden Beweiserleichterungen ähneln sich durch das Abstellen auf Erfahrungssätze der Lebenserfahrung und auf Wahrscheinlichkeiten. Wegen der Unklarheit der Herleitung und des Anwendungsbereichs der tatsächlichen Vermutung ${ }^{101}$ sowie der vorgeworfenen Nähe zum Anscheinsbeweis lassen sich die beiden Beweiserleichterungen in ihren Voraussetzungen nicht klar von einander abtrennen. Die tatsächliche Vermutung hat jedoch mit der Beweislastumkehr eine stärkere Rechtsfolge, sodass aufgrund der ähnlichen Voraussetzungen festgehalten werden kann, dass an die Anerkennung einer tatsächlichen Vermutung höhere Anforderungen als an das Anerkennen eines Anscheinsbeweises zu stellen sind.

\section{Sekundäre Darlegungslast}

Die Darlegungslast, auch Behauptungslast genannt, ${ }^{102}$ regelt, welche Par- 792 tei eine Behauptung aufstellen muss, um prozessuale Nachteile zu vermeiden ${ }^{103}$ Die Notwendigkeit begünstigende Tatsachen selbst vorzutragen, ergibt sich aus der Verhandlungsmaxime ${ }^{104}$ Behauptungs- und Beweislast stimmen grundsätzlich überein ${ }^{105}$ sodass jede Partei für sie begünstigende Umstände zu behaupten und zu beweisen hat ${ }^{106}$ Ausnahmsweise kann sich jedoch eine sekundäre Darlegungslast, auch Substantiierungslast genannt. ${ }^{107}$ ergeben.

99 Leipold, in: Stein/Jonas ${ }^{22}$, § 286 ZPO Rn. 139; Reichold, in: Thomas/Putzo ${ }^{34}$, § 286 ZPO Rn. 13.

100 Leipold, in: Stein/Jonas ${ }^{22}$, § 286 ZPO Rn. 139.

101 Laumen, in: Baumgärtel ${ }^{2}, \S 14$ Rn. 1.

102 Paulus ${ }^{4}$, Rn. 413; Prütting, in: MüKo-ZPO $^{4}$, § 286 Rn. 134.

103 Jauernig/Hess ${ }^{30}, \S 50$ Rn. 1.

104 Laumen, in: Prütting/Gehrlein ${ }^{5}$, § 286 ZPO Rn. 70.

105 Jauernig/Hess ${ }^{30}, \S 50$ Rn. 1.

106 Oben Rn. 772

107 Laumen, in: Prütting/Gehrlein ${ }^{5}$, § 286 ZPO Rn. 73. 
793 Voraussetzung für eine solche sekundäre Darlegungslast ist, dass die beweisbelastete Partei Informationen nicht hat oder deren Beschaffung unzumutbar ist 108 Dafür reicht es noch nicht aus, dass es der einen Partei wesentlich schwerer fällt als der anderen Partei 109 Sie muss vielmehr alles Mögliche und Zumutbare tun, damit von der Gegenpartei eine Aufklärung verlangt werden kann ${ }^{110}$ Die Gegenpartei hingegen muss genaue Kenntnis von diesen bestimmten rechtserheblichen Tatsachen haben oder ihr muss die Beschaffung der Informationen leicht sein und ihr muss ein Vortrag zu den Tatsachen zumutbar sein ${ }^{111}$ Die Informationen müssen sich auf Vorgänge im Wahrnehmungsbereich der Gegenpartei beziehen ${ }^{112}$ Allein die Tatsache, dass die Darlegung der beweisbelasteten Partei wesentlich schwerer fällt als der Gegenpartei, reicht nicht aus 113

794 Die Partei mit der Darlegungslast muss lediglich pauschal eine Tatsache behaupten ${ }^{114}$ für die jedoch eine gewisse Wahrscheinlichkeit sprechen muss ${ }^{115}$ Die Gegenpartei muss anschließend durch ein substantiiertes Vorbringen diese Behauptung bestreiten ${ }^{116}$ Falls ihr dies nicht gelingt, gelten die Behauptungen der beweisbelasteten Partei als nach $\S 138$ Abs. 3 ZPO zugestanden 117 Wenn die Partei es jedoch ohne überzeugenden Grund unterlässt einen Beweis zu erbringen, obwohl nur sie über die Möglichkeit verfügt, spricht die Lebenserfahrung dafür, dass ein solcher nicht vorhanden sind ${ }^{118}$ Der Umfang des substantiierten Bestreitens hängt vom Wechselspiel zwischen Vortrag und Gegenvortrag ab ${ }^{119}$ Gelingt hingegen das substantiierte Bestreiten, geht die Beweislast nicht über ${ }^{120}$ Die beweisbe-

108 BGH, Urteil v. 7. 12. 1998, II ZR 266/97 - BGHZ 140, 156, 158; Leipold, in: Stein/ Jonas $^{22}$, § 138 ZPO Rn. 37.

$109 B G H$, Urteil v. 17. 10. 1996, IX ZR 293/95 - NJW 1997, 128, 129.

110 Musielak, in: FG 50 Jahre BGH, Bd. 3, 193, 197.

$111 B G H$, Urteil v. 1.12.1982, VIII ZR 279/81 - BGHZ 86, 23, 29; Musielak, Grundkurs $^{11}$, Rn. 403; Prütting, in: MüKo-ZPO ${ }^{4}$ \& 286 Rn. 103; Reichold, in: Thomas/Putzo ${ }^{34}$, Vorbem $§ 284$ ZPO Rn. 18.

112 Leipold, in: Stein/Jonas ${ }^{22}$, § 138 ZPO Rn. 37.

113 Ebd. \$ 138 ZPO Rn. 37.

114 Laumen, in: Prütting/Gehrlein ${ }^{5}$, § 286 ZPO Rn. 73; Jauernig/Hess ${ }^{30}$, § 50 Rn. 3.

115 Musielak, Grundkurs ${ }^{11}$, Rn. 403.

116 Laumen, in: Prütting/Gehrlein ${ }^{5}$, § 286 ZPO Rn. 73; Jauernig/Hess ${ }^{30}$, § 50 Rn. 3.

117 Jauernig/Hess ${ }^{30}$, § 50 Rn. 3; Musielak, Grundkurs ${ }^{11}$, Rn. 403; Prütting, in: MüKo$\mathrm{ZPO}^{4}$ \& 286 Rn. 103.

118 Musielak, Grundkurs ${ }^{11}$, Rn. 403.

119 Prütting, in: MüKo-ZPO ${ }^{4}$ \& 286 Rn. 136.

120 Leipold, in: Stein/Jonas ${ }^{22}$, § 138 ZPO Rn. 38. 
lastete Partei muss dann beweisen, dass die beim substantiierten Bestreiten vorgebrachten Tatsachen nicht zutreffen 121

\section{Schutzbehauptungen durch freie richterliche Beweiswürdigung verhin- dern}

Die Notwendigkeit für Beweiserleichterungen wird dadurch begründet, dass sich der Account-Inhaber ohne solche durch eine einfache Behauptung vom Vertrag lösen könnte. Eine Alternative zur Beweiserleichterung besteht jedoch darin, dass diese Schutzbehauptung als solche entlarvt wird und unberücksichtigt bleibt. Über die freie richterliche Beweiswürdigung ( $\$ 286$ Abs. 1 S. 1 ZPO) können solche Schutzbehauptungen verhindert werden 122 Wird eine Behauptung spät vorgetragen, beispielsweise erst im Prozess, so kann ein Richter diese als Schutzbehauptung entlarven und unberücksichtigt lassen ${ }^{123}$ Bei der Zurückweisung von Schutzbehauptungen besteht das allgemeine Problem, dass ein Richter nicht gegen den Anspruch auf rechtliches Gehör verstoßen darf (Art. 103 Abs. 1 GG). Erhebt er bezüglich Ausführungen einer Partei, die er als Schutzbehauptung ansieht, keinen Beweis, verstößt er gegen den Anspruch der behaupteten Partei auf rechtliches Gehör 124 Erhebt er jedoch Beweis bezüglich der Behauptung kann er im Rahmen seiner freien Beweiswürdigung zum Schluss kommen, dass es sich um eine Schutzbehauptung handelt.

In der Rechtsprechung lassen sich Fälle finden, bei denen eindeutig keine Schutzbehauptungen vorliegen. Das $L G$ Köln hat im Rahmen der freien Beweiswürdigung einem Besitzer eines Imbissanhängers geglaubt, dass ihm ein übler Streich gespielt wurde, als der Anhänger auf einer InternetAuktionsplattform zum Verkauf angeboten wurde, obwohl der Besitzer den Wagen auf weiteren Vergnügungsmärkten einzusetzen beabsichtigte ${ }^{125}$ Beispielsweise konnte sich das $L G$ Kassel davon überzeugen, dass der Enkel und nicht dessen 82-jährige Großmutter, die glaubhaft über keine techni-

$121 B A G$, Urteil v. 12.8. 1976, 2 AZR 237/75 - NJW 1977, 167; LG Düsseldorf, Urteil v. 21.3. 2012, 12 O 579/10 - NJW 2012, 3663, 3663 f.; Leipold, in: Stein/Jonas ${ }^{22}$, $\S 138$ ZPO Rn. 38.

122 So auch KG Berlin, Urteil v. 10. 3. 2005, 8 U 122/04 - MDR 2005, 1431.

123 Siehe OLG Hamm, Beschluss v. 22. 8. 2006, 2 Ss OWi 528/06 - NZV 2007, 96, 97.

124 Vgl. KG Berlin, Urteil v. 10.3. 2005, 8 U 122/04 - MDR 2005, 1431.

125 LG Köln, Urteil v. 27. 10.2005, 8 O 15/05 - BeckRS 2006, 07259. 
schen Kenntnisse verfügt, den auf die Großmutter lautenden Account verwendet hat 126

797 Im Gegensatz lassen sich Schutzbehauptungen nach einer Abwägungen der Umstände im Einzelfall auch entlarven. Die Annahme einer Schutzbehauptung liegt beispielsweise nahe, wenn ein Anschlussinhaber behauptet, nicht er sondern seine minderjährige Tochter hätte pornographische Inhalte über den Bildschirmtext-Anschluss abgerufen ${ }^{127}$ Ein Indiz für eine Schutzbehauptung kann ebenfalls sein, wenn der Anschluss-Inhaber eines Internetanschlusses behauptet, der illegale Download von Musik sei durch seinen dreizehnjährigen Sohn ohne Wissen des Anschlussinhabers erfolgt, sich jedoch auf dem Computer des Sohns ein Ordner namens „Papas Music“ befindet, in dem Musik gespeichert ist, die von Jugendlichen im Alter des Sohns regelmäßig nicht gehört wird 128

798 Die Umstände des Einzelfalls können auch in weiteren Fällen auf Schutzbehauptungen hindeuten. Ein spätes Bestreiten der Abgabe oder das späte Behauptung eines Missbrauchs können Indizien für eine Schutzbehauptung sein. Ein vorheriger Kontakt mit dem Geschäftsgegner, bei dem die später erfolgte Erklärung angekündigt wurde, kann bei späterem Bestreiten ein Indiz für eine Schutzbehauptung sein. Wenn durch reihenweises Bestreiten verschiedener Tatsachen an der Glaubwürdigkeit dieser Behauptungen ernsthafte Zweifel bestehen, kann ein Gericht dadurch Schutzbehauptungen erkennen. In einem Fall glaubte das AG München dem Beklagten nicht 129 Er hatte behauptet, dass er die Willenserklärung über den Account nicht abgegeben habe und dass er den Account nicht erstellt habe. Darüber hinaus bestritt er den Empfang für ein Dokument, dessen Empfang er schriftlich quittiert hatte. Wegen dieser Lüge wertete das AG München die anderen Behauptungen auch als Schutzbehauptungen ${ }^{130}$ Dies zeigt, dass die freie richterliche Beweiswürdigung durchaus in der Lage ist, durch die Gesamtheit des Vortrags einer Partei und deren Glaubwürdigkeit Schutzbehauptungen zu erkennen und deren Auswirkungen zu verhindern. Weitere Indizien für Schutzbehauptungen liegen vor, wenn die Willenserklärung mittels einer IP-Adresse abgegeben wurde, die geographisch dem Bereich des AccountInhabers zuzuordnen und auf seinen Internet-Anbieter registriert ist. Wei-

126 LG Kassel, Urteil v. 15. 4. 2008, 9 O 2539/06 - NJW-RR 2009, 781.

127 Vgl. OLG Oldenburg, Urteil v. 11. 1. 1993, 13 U 133/92 - NJW 1993, 1400.

128 OLG Köln, Urteil v. 23. 3. 2012, 6 U 67/11 - MMR 2012, 387, 389.

129 AG München, Urteil v. 24. 4. 2007, 161 C 24310/05 - CR 2007, 816, 817.

130 Ebd. 817. 
tere Indizien können sein, dass in einem Text Spezialwissen oder für den Account-Inhaber typische Ausdrücke verwendet wurden.

Die Möglichkeit, Schutzbehauptungen über die freie richterliche Beweiswürdigung zu entlarven, zeigt, dass das Problem nicht notwendigerweise über Beweiserleichterungen gelöst werden muss. Für den Geschäftsgegner sind Beweiserleichterungen jedoch vorteilhafter, weil die Situation für die Schutzbehauptungen umgedreht wird. Der Geschäftsgegner muss grundsätzlich beweisen, dass eine Erklärung vom Account-Inhaber stammt und gegebenenfalls, dass sein Bestreiten eine Schutzbehauptung darstellt. Bei einer Beweiserleichterung muss der Account-Inhaber darlegen oder beweisen, dass er die Erklärung nicht abgegeben hat.

\section{Anerkannte Beweiserleichterungen in ähnlichen Konstellationen}

Nachfolgend sollen zunächst anerkannte Beweiserleichterungen von Fällen, die dem Missbrauch von Zugangsdaten im Internet ähnlich sind, betrachtet werden, um anschließend Schlussfolgerungen für Beweiserleichterung bei Missbrauch von anderen Zugangsdaten im Internet zu ziehen.

\section{Elektronische Signatur}

Bei der elektronischen Signatur ${ }^{131}$ hat der Gesetzgeber die Beweiserleichte- 801 rung gesetzlich normiert. Der Beweiswert von qualifizierten elektronischen Signaturen ist in $\S 371$ a Abs. 1 S. 2 ZPO, der $\S 292$ a ZPO a.F. ablöst.132 geregelt. Bei der Regelung handelt es sich um einen gesetzlich normierten Anscheinsbeweis ${ }^{133}$ Es entsprach dem gesetzgeberischen Willen, die allgemeinen richterrechtlichen Voraussetzungen für einen Anscheinsbeweis für diesen Einzelfall zu kodifizieren ${ }^{134}$ Teilweise wird bezweifelt, dass dies gelungen ist ${ }^{135}$ Erfahrungssätze bei der elektronischen Signatur lägen nicht

131 Dazu oben Rn.73 ff.

132 Musielak, in: FG Vollkommer, 237, 249.

133 Reichold, in: Thomas/Putzo ${ }^{34}, \S 371$ a ZPO Rn. 2; A. Stadler, ZZP 115 (2002), 413, 432; W. Zimmermann, in: MüKo-ZPO 4 \& 371a Rn. 4.

134 Begr. FormAnpG, BT-Drucks. 14/4987, S. 23; Begr. JKomG, BT-Drucks. 15/4067, S. 34; Huber, in: Musielak ${ }^{10}$, § 371a ZPO Rn. 6.

135 Huber, in: Musielak ${ }^{10}, \S 371$ a ZPO Rn. 7. 
vor, sodass die notwendige Vermutungsbasis fehle ${ }^{136}$ Dagegen lässt sich jedoch einwenden, dass in diesem Fall ein Anscheinsbeweis ohne ersten Anschein ${ }^{137}$ ohne vorhandene Erfahrungssätze begründet werden könnte. Darüber hinaus wird gegen die Plausibilität der Vermutungsbasis eingebracht, dass eine Delegation der Signierung an Mitarbeiter zu erwarten sei ${ }^{138}$ Es liegt somit kein echter Anscheinsbeweis vor, sondern vielmehr eine gesetzliche Beweisregel ${ }^{139}$ Der Beweis des Vorliegens der Voraussetzungen von $\S 371 \mathrm{a}$ Abs. 1 S. 2 ZPO ist bei qualifizierten elektronischen Signaturen von Anbietern ohne Akkreditierung schwer zu führen ${ }^{140}$ Die einfache elektronische Signatur und die fortgeschrittene elektronische Signatur sind von $\S 371$ a Abs. 1 S. 2 ZPO nicht erfasst ${ }^{141}$ Für fortgeschrittene elektronische Signaturen ist ein Anscheinsbeweis nach den richterrechtlichen Grundsätzen je nach konkreter technischer Ausgestaltung jedoch möglich ${ }^{142}$

802 Hinter dieser Kodifizierung des Anscheinsbeweises steckt der Sphärengedanke. Der Erklärungsempfänger kann zu den Umständen der Abgabe einer elektronisch signierten Erklärung nicht substantiiert vortragen ${ }^{143}$ Dem Erklärungsempfänger wird somit der Beweis von Tatsachen erleichtert, die er kaum beweisen kann. Doch bei qualifizierten elektronischen Erklärungen besteht das Problem, dass es schwierig ist, den Beweis über lange zurückliegende Erklärungen zu führen 144 Teilweise wird einschränkend vertreten, dass der Anscheinsbeweis des § 371a Abs. 1 S. 2 ZPO nur dann zum Tragen kommt, wenn feststeht, dass der Schlüssel-Inhaber die Erklärung selbst signiert hat ${ }^{145}$ Dagegen spricht jedoch, dass die eigenhändige Signierung kein konstitutives Merkmal der qualifizierten elektronischen Signatur ist ${ }^{146} \S 2$ Nr. 2 lit. c SigG setzt nach seinem Wortlaut nur voraus, dass der

136 Schemmann, ZZP 118 (2005), 161, 181; A. Stadler, ZZP 115 (2002), 413, 434.

137 Oben Rn. 788

138 Schemmann, ZZP 118 (2005), 161, 182; dagegen Musielak, in: FG Vollkommer, 237, $249 \mathrm{f}$.

139 Berger, in: Stein/Jonas ${ }^{22}$, § 371a ZPO Rn. 15; W. Zimmermann, in: MüKo-ZPO ${ }^{4}$ § 371a Rn. 4; Czeguhn, JuS 2004, 124, 126.

140 Roßnagel, MMR 2000, 451, $459 \mathrm{f}$.

141 Huber, in: Musielak ${ }^{10}$, § 371a ZPO Rn. 3; Trautwein, in: Prütting/Gehrlein ${ }^{5}$, § 371a ZPO Rn. 2.

142 Ernst, MDR 2003, 1091, 1092.

143 Trautwein, in: Prütting/Gehrlein ${ }^{5}, \S 371$ a ZPO Rn. 5.

144 Vgl. Roßnagel, MMR 2002, 215, $218 \mathrm{f}$.

145 Jandt, K\&R 2009, 548, 554.

146 Anders jedoch ebd. 550 f. In diese Richtung auch Roßnagel, MMR 2008, 22, 27. 
Schlüssel-Inhaber die Zugangsdaten grundsätzlich ,unter seiner alleinigen Kontrolle halten kann", nicht dass er es im konkreten Fall auch getan hat. Dies entspricht auch dem Willen des Gesetzgebers, nach dem $\S 2$ Nr. 2 lit. c SigG sicherstellt, dass der Schlüssel-Inhaber ,seine Signaturerstellungseinheit vor unbefugter Nutzung schützen können muss. ${ }^{147}$ Demnach reicht die Möglichkeit des Schutzes. Dass der Schlüssel-Inhaber die Signaturerstellungseinheit auch im konkreten Einzelfall gegen unbefugte Nutzung geschützt hat, ist gerade nicht erforderlich. § 371a Abs. 1 S. 2 ZPO soll nach dem Normzweck gerade auch die Fälle erfassen, bei denen nicht geklärt werden kann, wer die Erklärung elektronisch signiert hat ${ }^{148}$

Der Wortlaut des $\S 371$ a Abs. 1 S. 2 ZPO verwendet die richterrechtliche Voraussetzung des Widerlegens des Anscheinsbeweises mit dem Rückgriff auf das Erschüttern 149 durch Tatsachen, die ernstliche Zweifel an der Vermutungsbasis begründen. Welche Anforderungen an das Erschüttern zu stellen sind, ist unklar. Teilweise wird behauptet, es dürfen keine zu hohen Anforderungen gestellt werden ${ }^{150}$ Andererseits wird behauptet, der Zweck, den sicheren Rechtsverkehr durch qualifizierte elektronische Signaturen zu gewähren, soll durch hohe Anforderungen an das Erschüttern erreicht werden ${ }^{151}$ Zum Erschüttern reichen jedenfalls rein theoretische Möglichkeiten alternativer Geschehensabläufe nicht aus ${ }^{152}$ Das Erschüttern der Vermutungsbasis des $\S 371$ a Abs. 1 S. 2 ZPO ist schwerer als das Erschüttern der Vermutungsbasis bei Einsatz einer ec-Karte ${ }^{153}$, weil der Missbrauch regelmäßig später auffallen wird und dadurch die Beweisführung erschwert wird 154 Dadurch lässt dieser Anscheinsbeweis den Schlüssel-Inhaber das Missbrauchsrisiko tragen ${ }^{155}$ Problematisch ist, dass die Sicherheitsanforderungen fest ins Gesetz geschrieben sind, sodass der Anscheinsbeweis mit neuen Erkenntnissen im Wege der richterlichen Würdigung keiner neuen Bewertung unterworfen werden kann. Dadurch werden die Systemrisiken

147 Begr. SigG, BT-Drucks. 14/4662, S. 18.

148 Huber, in: Musielak ${ }^{10}$, § 371a ZPO Rn. 6; Schemmann, ZZP 118 (2005), 161, 167.

149 Oben Rn. 790

150 Armgardt/Spalka, K\&R 2007, 26, 29; Bergfelder, S. 308; Greger, in: Zöller ${ }^{30}$, § 371a ZPO Rn. 2.

151 Trautwein, in: Prütting/Gehrlein ${ }^{5}, \S 371$ a ZPO Rn. 6.

152 Greger, in: Zöller ${ }^{30}$, § 371a ZPO Rn. 2.

153 Dazu unten Rn. 812 ff.

154 Borges, Verträge, S. 509.

155 Berger, in: Stein/Jonas ${ }^{22}$, § 371a ZPO Rn. 6. 
der qualifizierten elektronischen Signatur dem Schlüssel-Inhaber aufgebür$\operatorname{det} 156$

804 Das Erschüttern der Vermutungsbasis $§ 371$ a Abs. 1 S. 2 ZPO ist in folgenden Fällen denkbar. Kann der Schlüssel-Inhaber beweisen, dass ein Dritter die Erklärung signiert hat, entfällt die Vermutungsbasis, die sich nur auf die Echtheit der Erklärung bezieht ${ }^{157}$ Dazu muss der Schlüssel-Inhaber nur vortragen, dass er die Chip-Karte samt der PIN weitergeben hat 158 Anders war noch die Regelung des § 292a ZPO a.F., wonach sich die Vermutungsbasis auf die Abgabe mit Willen des Schlüssel-Inhabers bezog. Den Anscheinsbeweis des § 292a ZPO a.F. konnte der Schlüssel-Inhaber somit nicht durch die Weitergabe der Chip-Karte erschüttern. Der Anscheinsbeweis des $\S 371$ a Abs. 1 S. 2 ZPO hingegen ist bereits erschüttert, wenn der Schlüssel-Inhaber die Chip-Karte aus der Hand gegeben hat. Ferner kann der Schlüssel-Inhaber die Vermutungsbasis dadurch erschüttern, dass ihm ein anderes Dokument, als jenes, das er signieren wollte, untergeschoben wurde ${ }^{159}$ Dies kann durch einen Angriff mittels Trojaner ${ }^{160}$ auf die Software zur Erstellung der Signatur erfolgen ${ }^{161}$ Der Schlüssel-Inhaber muss dann darlegen, dass der Rechner infiziert war, sodass die externe Manipulation möglich war ${ }^{162}$ Ebenso kann der Beweis des Diebstahls der Signaturkarte sowie des Ausspähens der PIN die Vermutungsbasis erschüttern 163 Vereinzelt wird behauptet, dass wegen des stets möglichen Einsatzes von Trojanern der Anscheinsbeweis schon erschüttert sei, wenn der betreffende Rechner des Schlüssel-Inhabers mit dem Internet oder anderen Rechnern verbunden ist 164

805 Darüber hinaus können Fehler bei der Überprüfung der Identitätsbehauptung des Schlüssel-Inhabers beim Ausstellen des Signaturschlüssels auftre-

156 Borges, Verträge, S. 509 f.

157 Bergfelder, S. 309; Berger, in: Stein/Jonas ${ }^{22}$, § 371a ZPO Rn. 17.

158 Berger, in: Stein/Jonas ${ }^{22}$, § 371a ZPO Rn. 17; Huber, in: Musielak ${ }^{10}$, § 371a ZPO Rn. 10; Schemmann, ZZP 118 (2005), 161, 174.

159 Huber, in: Musielak $^{10}$, § 371a ZPO Rn. 10; Schemmann, ZZP 118 (2005), 161, 173; Roßnagel/Fischer-Dieskau, NJW 2006, 806, 807.

160 Oben Rn. 193

161 Armgardt/Spalka, K\&R 2007, 26, 29.

162 Gassen, S. 245.

163 Berger, in: Stein/Jonas ${ }^{22}$, § 371a ZPO Rn. 17; Bergfelder, S. 311; Redeker, ITRecht $^{5}$, Rn. 915; Schemmann, ZZP 118 (2005), 161, 173; W. Zimmermann, in: MüKo-ZPO 4 \& 371a Rn. 4.

164 Armgardt/Spalka, K\&R 2007, 26, 32. 
ten. Kann der vermeintliche Schlüssel-Inhaber diese beweisen, wird dadurch die Vermutungsbasis erschüttert ${ }^{165}$ Der Behauptung, dass dies kaum vorkommen werde ${ }^{166}$ ist zu widersprechen. Insbesondere durch die Lockerung der Anforderungen an die Identifizierung ${ }^{167}$ ist das Erstellen einer qualifizierten elektronischen Signatur auf einen fremden Namen einfacher möglich geworden. Die technische Unsicherheit des Systems kann ebenfalls die Vermutungsbasis erschüttern. Beim Beweis eines Algorithmenverfalls, also der Möglichkeit den privaten Schlüssel anhand des öffentlichen Schlüssels mit geringem Aufwand zu erreichen ${ }^{168}$ ist die Vermutungsbasis ebenfalls erschüttert 169

Scheitert der Anscheinsbeweis des $\S 371$ a Abs. 1 S. 2 ZPO daran, dass der Schlüssel-Inhaber die Vermutungsbasis erschüttert, kann sich dessen Haftung aus dem materiellen Recht ergeben 170 Nach den allgemeinen Rechtsscheingrundsätzen haftet der Schlüssel-Inhaber unter gewissen Umständen für den Missbrauch seines qualifizierten elektronischen Signaturschlüssels ${ }^{171}$ So kann der Schlüssel-Inhaber zwar durch die Weitergabe der Zugangsdaten und die anschließende Signierung durch einen Dritten die Vermutungsbasis der Echtheit der Erklärung zerstören. Bei einer qualifizierten elektronischen Signatur wird ihm die Erklärung des Dritten dabei jedoch nach Rechtsscheingrundsätzen zugerechnet.

Aus der Betrachtung der gesetzlich normierten Beweiserleichterung des $\S 371 \mathrm{a}$ Abs. 1 S. 2 ZPO lassen sich grundsätzliche Wertungen herausarbeiten. Zum einen ist es für den Anscheinsbeweis entscheidend, dass das Authentisierungsverfahren einen hohen Sicherheitsstandard aufweist. Die qualifizierte elektronische Signatur reicht nach dem gesetzgeberischen Willen dafür aus ${ }^{172}$ Die Sicherheit der elektronischen Signatur zeichnet sich durch zwei Merkmale aus. Erstens setzt sie auf eine Zwei-Faktor-Authentisierung ${ }^{173}$ Für eine erfolgreiche Authentisierung ist neben dem Wissen des PIN der Besitz der physisch einmaligen Chip-Karte erforderlich,

165 Schemmann, ZZP 118 (2005), 161, 172.

166 Dästner, NJW 2001, 3469.

167 Unten Rn. 887

168 Oben Rn. 80

169 Schemmann, ZZP 118 (2005), 161, 175.

170 Berger, in: Stein/Jonas ${ }^{22}$, § 371a ZPO Rn. 18.

171 Unten Rn. 882 ff.

172 Begr. FormAnpG, BT-Drucks. 14/4987, S. 23.

173 Unten Rn. 883 . 
was einen Missbrauch erheblich erschwert 174 Zweitens muss die Identität des Schlüssel-Inhabers bei Ausstellung des Zertifikats zuverlässig überprüft werden ${ }^{175}$ Aus der Wertung des $\S 371$ a Abs. 1 S. 2 ZPO kann daher abgeleitet werden, dass für ähnlich sichere Authentisierungsverfahren bei einer unveränderten Lage von Missbrauchsmöglichkeiten im Vergleich zum Zeitpunkt der gesetzgeberischen Entscheidung ein Anscheinsbeweis in Betracht kommt. Zum anderen lässt sich aus der Gesetzesbegründung zu § 371a Abs. 1 S. 2 ZPO entnehmen, dass die Schutzwürdigkeit des Geschäftsgegners und das Sicherstellen des Vertrauens des Marktes in die elektronische Signatur, bei der Erwägung eines Anscheinsbeweises eine Rolle spielen können 176

\section{Bildschirmtext (Btx)}

808 Beim Bildschirmtex ${ }^{177}$ wurden Beweiserleichterungen herrschend angenommen. Nur vereinzelt wurden sie abgelehnt ${ }^{178}$ Gegen diese Beweiserleichterung wird eingebracht, dass sich der Authentisierungsnehmer die $\mathrm{Au}-$ thentisierungsmethoden auswählen könnte ${ }^{179}$ Ferner fehlten dem AccountInhaber positive sowie negative Nachweismöglichkeiten des elektronischen Vertragsschlusses ${ }^{180}$ Eine Beweiserleichterung könnte sich nur aus der Beweisnot der anderen Partei ergeben ${ }^{181}$ Dies verbiete sich jedoch bei den in Frage stehenden Erfüllungsansprüchen, weil bei diesen der Geschäftsgegner einen sicher beweisbaren Vertragsschluss herbeiführen könnte ${ }^{182}$ und weil er die negative Tatsache, dass er den Vertragsschluss nicht herbeigeführt habe, nur schwer beweisen könne 183

809 Beweiserleichterungen werden jedoch in unterschiedlicher Form angenommen. Überwiegend wird eine tatsächliche Vermutung beim Missbrauch

\footnotetext{
174 Oben Rn. 584

175 Unten Rn. 886

176 Vgl. Begr. JKomG, BT-Drucks. 15/4067, S. 34.

$177 \mathrm{Zu}$ dessen technischen Grundlagen oben Rn. 498

178 Auerbach, CR 1988, 18; Clemens, NJW 1985, 1998, 2005; Kleier, WRP 1983, 534, 536.

179 Auerbach, CR 1988, 18.

180 Clemens, NJW 1985, 1998, 2005.

181 Kleier, WRP 1983, 534, 536.

182 Ebd. 536. Zu alternativen Sicherungsmöglichkeiten oben Rn. 657

183 Kuhn, S. 255.
} 
des Bildschirmtext-Anschlusses angenommen ${ }^{184}$ Dabei wird die tatsächliche Vermutung wie hier ${ }^{185}$ als Beweislastumkehr verstanden ${ }^{186}$ Die Sicherung durch die in das Endgerät eingespeicherte Anschlussziffer sowie das Passwort begründen diese Vermutung ${ }^{187}$ Die tatsächliche Vermutung bestehe darin, dass eine missbräuchliche Nutzung des Bildschirmtext-Anschlusses nur durch Zutun des Account-Inhabers ermöglicht wurde ${ }^{188}$ Den Inhalt des Vertrags müsse jedoch der Geschäftsgegner beweisen ${ }^{189}$ Durch die Notwendigkeit das spezielle Bildschirmtext-Gerät des Anschlussinhabers zu verwenden, ist ein Missbrauch nur in der räumlichen Sphäre des Account-Inhabers möglich, sodass dieser den Missbrauch gut kontrollieren kann 190

In der Literatur wird hingegen häufig die schwächere Form der Beweiserleichterung in Form des Anscheinsbeweises bevorzugt ${ }^{191}$ Das Vorliegen des Anscheinsbeweises wird entweder durch das Vorliegen des Regelfalls, dass berechtigt gehandelt wurde $\sqrt{192}$ oder durch die hinreichende Sicherung des Authentisierungsvorgangs ${ }^{193}$ begründet. Ein Beweis der Vermutungsbasis sei dabei jedoch nur bei nicht manipulierbaren Aufzeichnungen möglich ${ }^{194}$ Teilweise wird dieser Anscheinsbeweis auf Sekundäransprüche beschränkt 195

Aus den Beweiserleichterungen beim Bildschirmtext lässt sich die Erkenntnis gewinnen, dass eine starke Beweiserleichterung wie die tatsäch-

184 OLG Köln, Urteil v. 30. 4. 1993, 19 U 134/92 - CR 1993, 552; Urteil v. 21. 11. 1997, 19 U 128/97 - NJW-RR 1998, 1277, 1279; OLG Oldenburg, Urteil v. 11. 1. 1993, 13 U 133/92 - NJW 1993, 1400, 1406; Borsum/Hoffmeister, BB 1983, 1441, 1446; dies., NJW 1985, 1205, 1207; Brinkmann, BB 1981, 1183, 1187.

185 Oben Rn.781

186 OLG Oldenburg, Urteil v. 11.1. 1993, 13 U 133/92 - NJW 1993, 1400, 1406, nicht explizit, aber durch den Verweis auf die $B G H$-Entscheidung eindeutig: $B G H$, Urteil v. 18. 9. 1984, VI ZR 223/82 - BGHZ 92, 143, $146 \mathrm{f}$.

187 OLG Köln, Urteil v. 21. 11. 1997, 19 U 128/97 - NJW-RR 1998, 1277, 1279; OLG Oldenburg, Urteil v. 11. 1. 1993, 13 U 133/92 - NJW 1993, 1400, 1406.

188 OLG Köln, Urteil v. 21. 11.1997, 19 U 128/97 - NJW-RR 1998, 1277, 1279; Borsum/Hoffmeister, BB 1983, 1441, 1446; dies., NJW 1985, 1205, 1207.

189 Borsum/Hoffmeister, BB 1983, 1441, 1446; dies., NJW 1985, 1205, 1207.

190 OLG Köln, Urteil v. 30. 4. 1993, 19 U 134/92 - CR 1993, 552.

191 Kuhn, S. 253; Probandt, UFITA 98 (1984), 9, 18.

192 Probandt, UFITA 98 (1984), 9, 18.

193 Kuhn, S. 253.

194 Ebd. S. 253.

195 Ebd. S. $254 \mathrm{f}$. 
liche Vermutung bei einem sicheren Authentisierungsverfahren in Betracht kommt. Das Bildschirmtext-System setzt auf eine Zwei-Faktor-Authentisierung dadurch, dass zum Einwählen neben dem geheimen Kennwort die im Endgerät eingespeicherte Anschlusskennung notwendig ist 196 Die Besitzkomponente beim Bildschirmtext, die sich ebenfalls durch eine physische Einmaligkeit auszeichnet, ist jedoch besonders gesichert. Da es sich um ein großes und sperriges Gerät handelt, verlässt es regelmäßig nicht die räumliche Sphäre des Anschlussinhabers. Im Gegensatz zu einer Chip-Karte, die der Inhaber im Portemonnaie stets mit sich tragen kann, kann der Anschlussinhaber das in seiner räumlichen Sphäre befindliche BildschirmtextGerät besser kontrollieren. Da ein Teilen des Bildschirmtext-Anschlusses in der häuslichen Gemeinschaft zu erwarten ist ${ }^{197}$ bezieht sich die Beweiserleichterung nur darauf, dass die Nutzung des Anschlusses nur mit Zutun des Anschlussinhabers ermöglicht wurde ${ }^{198}$ Ferner zeigt die Diskussion um die Beweiserleichterungen, dass die Grenzen zwischen einem Anscheinsbeweis und einer tatsächlichen Vermutung schwer zu ziehen sind 199

\section{3. ec-Karte}

812 Der Anscheinsbeweis beim Missbrauchs von ec-Karte, der auf Kreditkarten ebenso angewendet wird, bietet in Rechtsprechung und Literatur ausführliches Anschauungsmaterial. Einige Stimmen aus Literatur und Rechtsprechung meinen, dass die am 31.10.2009 in Kraft getretene Neuregelung in $\S 675$ w S. 3 BGB die Beibehaltung des Anscheinsbeweises verbiete 200 Überwiegend wird jedoch gestützt auf den Willen des Gesetzgebers ${ }^{201}$ angenommen, dass die Neuregelung keine Veränderung der Rechtslage bezweckte und der entwickelte Anscheinsbeweis weiterhin angewendet werden kann 202 Unabhängig davon, ob der Anscheinsbeweis in seiner alten

\footnotetext{
196 Oben Rn. 498

197 Oben Rn. 300

198 OLG Oldenburg, Urteil v. 11. 1.1993, 13 U 133/92 - NJW 1993, 1400, 1401.

199 Dazu bereits abstrakt oben Rn. 791 .

200 So im Ergebnis AG Berlin Mitte, Urteil v. 25. 11. 2009, 21 C 442/08 - NJW-RR 2010, 407, 408; Franck/Massari, WM 2009, 1117, 1126; Scheibengruber, BKR 2010, 15, $20 \mathrm{f}$.
}

201 BT-Drucks. 16/11643, S. 114.

202 LG Berlin, Urteil v. 22. 6. 2010, 10 S 10/09 - NJW-RR 2011, 352, 354; AG Frankfurt, Urteil v. 10. 11. 2010, 29 C 1461/10-85 - WM 2011, 496, 497; AG Hamburg, 
Form weiter Bestand hat, lassen sich aus der Diskussion um dessen Voraussetzungen wertvolle Schlüsse für einen Anscheinsbeweis beim Missbrauch von Zugangsdaten im Internet ziehen.

Wird mit einer ec-Karte unter Verwendung der korrekten Zugangsdaten Geld an einem Automaten abgehoben, besteht ein Anscheinsbeweis dafür, dass der Kartenbesitzer diese selbst vorgenommen hat oder dass ein Dritter nach Entwendung der Karte wegen des unsorgsamen Umgangs mit der PIN Kenntnis von dieser erlangen konnte und dieser das Geld abgehoben hat 203 Der Grund für die Anerkennung des Anscheinsbeweises ist, dass quasi nicht beweisbar ist, dass der Bankkunde oder ein Berechtigter gehandelt hat ${ }^{204}$ Der Anscheinsbeweis hat eine ausdifferenzierte Ausformung. Ist beispielsweise unstrittig, dass der Karteninhaber die Abhebung nicht selbst vorgenommen hat, bezieht sich der Anscheinsbeweis nur darauf, dass dieser die PIN zusammen mit der Karte aufbewahrt hat 205 Wird nicht die Originalkarte sondern eine Dublette verwendet, wie beim Skimming der Fall ${ }^{206}$ kommt der Anscheinsbeweis nicht in Betracht ${ }^{207}$ Weil die Bank die Vermutungsbasis vollständig beweisen muss, liegt die Beweislast der Verwendung der Originalkarte bei ihr 208

Der Bankkunde kann die Vermutungsbasis des Anscheinsbeweises durch die konkrete Darlegung eines alternativen Geschehensablaufs erschüttern 209 Rein theoretische, alternative Möglichkeiten, die jedoch höchst unwahrscheinlich sind, reichen dafür nicht. Eine solche theoretische aber unwahrscheinliche Möglichkeit ist, dass ein Bankmitarbeiter als Innentäter

Urteil v. 28. 9.2010, 4 C 178/10 - WM 2011, 498; Bunte ${ }^{3}$, SB girocard Rn. 85; Casper/Pfeifle, WM 2009, 2343, 2347; Günther, WM 2013, 496, 497 f.; Grundmann, WM 2009, 1157, 1164; Maihold, in: Schimansky/Bunte/Lwowski ${ }^{4}$, § 54 Rn. 49, 109; Oechsler, WM 2010, 1381, 1382; Spindler, in: FS Nobbe, 215, 232 f.; v. Westphalen, in: Erman ${ }^{13}$, § 675w BGB Rn. 13.

$203 B G H$, Urteil v. 5. 10.2004, XI ZR 210/03 - BGHZ 160, 308, 314 ff.; Urteil v. 14. 11. 2006, XI ZR 294/05 - BGHZ 170, 18, Rn. 31; unbeanstandet von BVerfG, Beschluss v. 8. 12. 2009, 1 BvR 2733/06 - NJW 2011, 1129, Rn. 16.

204 Borges, Verträge, S. 496; Jungmann, in: Jahrbuch Junger Zivilrechtswissenschaftlicher 2007, 329, 331.

205 Jungmann, in: Jahrbuch Junger Zivilrechtswissenschaftlicher 2007, 329, 331.

206 Zum Skimming Schulte am Hülse/Welchering, NJW 2012, 1262, 1264.

207 BGH, Urteil v. 29. 11.2011, XI ZR 370/10 - NJW 2012, 1277, Rn. 16; Kollrus, MDR 2012, 377, 379; Günther, WM 2013, 496, 498; Maihold, in: Schimansky/ Bunte/Lwowski ${ }^{4}$, § 54 Rn. 116.

$208 B G H$, Urteil v. 29. 11.2011, XI ZR 370/10 - NJW 2012, 1277, Rn. 16.

209 Kollrus, MDR 2012, 377, 380. 
den Institutsschlüssel ausspäht oder die Sicherheitsinfrastruktur beeinträchtigt ${ }^{210}$ Ebenso theoretisch möglich, aber sehr unwahrscheinlich sei, dass der Dritte die mit 128-Bit verschlüsselten PIN errechnet hat ${ }^{211}$ Wegen der zunehmenden Rechenleistung moderner Computer sowie der Aggregation dieser in Botnetzen, die Hacker für solche Operationen nutzen können, wird daran gezweifelt, dass die 128-Bit-Verschlüsselung noch einen ausreichenden Schutz bietet 212 Dies zeigt, dass die Vermutungsbasis eines Anscheinsbeweises einer ständigen Überprüfung und Anpassung an neue Entwicklungen bedarf. Er unterliegt einer Dynamik, bei der die geänderte Lebenserfahrung das ständige Hinterfragen der Erfahrungssätze notwendig macht 213

815 Erschüttern kann der Karteninhaber den Anscheinsbeweis jedoch dadurch, dass er einen Diebstahl der Karte darlegt ${ }^{214}$ Dazu muss er nicht den Vollbeweis eines Diebstahls erbringen, sondern vielmehr nur äußere Umstände beweisen, die auf einen Diebstahl hindeuten. Dafür kommt es auf die Umstände des Einzelfalls an 215 Der Anscheinsbeweis kann ebenfalls dadurch erschüttert werden, dass eine konkrete Möglichkeit des Ausspähens der PIN bewiesen wird. Dies setze jedoch einen engen zeitlichen Zusammenhang zwischen dem Einsatz der ec-Karte mit dem späteren Missbrauch voraus ${ }^{216}$ Zwar wird der Ausspäher nach dem Diebstahl die ec-Karte schnell missbrauchen, um einer Sperrung zuvor zu kommen, bei anderen Konstellationen kann jedoch das Ausspähen und der Missbrauch Wochen oder Monate auseinander liegen ${ }^{217}$ Man kann daher an dem Erfordernis des zeitlichen Zusammenhangs zweifeln.

816 Eine Besonderheit des Anscheinsbeweises beim ec-Karten-Missbrauch besteht in der Begründung der Vermutungsbasis. Zwar lassen sich durchaus Erwägungen finden, die den typischen Geschehensablauf durch Empi-

210 BGH, Urteil v. 5. 10.2004, XI ZR 210/03 - BGHZ 160, 308, 318; Schulte am Hülse/Welchering, NJW 2012, 1262, 1265.

$211 B G H$, Urteil v. 5. 10.2004, XI ZR 210/03 - BGHZ 160, 308, 316; Bunte ${ }^{3}$, SB girocard Rn. 86.

212 Scheibengruber, BKR 2010, 15, 21.

213 Jungmann, in: Jahrbuch Junger Zivilrechtswissenschaftlicher 2007, 329, 354.

214 Hanau, Handeln unter fremder Nummer, S. 141; Bergfelder, S. 272.

215 Bergfelder, S. 272; Borges, Verträge, S. 502.

$216 B G H$, Urteil v. 5. 10. 2004, XI ZR 210/03 - BGHZ 160, 308, 317 f.; Hanau, Handeln unter fremder Nummer, S. 142 f.; ders., VersR 2005, 1215, 1219.

217 Schulte am Hülse/Welchering, NJW 2012, 1262, 1264. 
rie zu belegen oder widerlegen versuchen ${ }^{218}$ Dies verkennt jedoch, dass der Anscheinsbeweis ohne einen empirischen Befund über den Erfahrungssatz begründet wird 219 Weil kein Erfahrungssatz besteht, wird der Anscheinsbeweis vielmehr durch den Ausschluss alternativer Möglichkeiten begrün$\operatorname{det}{ }^{220}$ Es handele sich dabei um einen ,Anscheinsbeweis ohne ersten Anschein“ 221 Bei dieser Form durch Ausschluss alternativer Möglichkeiten gehört es jedoch zu der zu beweisenden Vermutungsgrundlage, dass die auszuschließenden Möglichkeiten, von der Bank bewiesen werden müssen 222 Dabei besteht jedoch das Problem, dass für die abschließende Bewertung der Möglichkeiten Auskünfte über die Sicherheit erforderlich sind, die jedoch die Sicherheit beeinträchtigen können 223

Die Annahme des Anscheinsbeweises bei der ec-Karte zeigt, dass die verwendete Zwei-Faktor-Authentisierung unter gewissen Umständen eine ausreichende Wahrscheinlichkeit für die Anerkennung der Beweiserleichterung darstellt. Dass der Anscheinsbeweis nur beim Einsatz der Originalkarte und nicht einer Dublette in Betracht kommt, zeigt, dass die physische Einmaligkeit der Besitz-Komponenten entscheidend ist. Sie erlaubt dem KartenInhaber Missbrauchsmöglichkeiten zu entdecken. Ist ihm die Karte abhandengekommen, beispielsweise beim Diebstahl des Portemonnaies, kann er die Gefahr eines Missbrauchs entdecken und durch Sperrung der Karte verhindern. Unausgesprochen, aber dennoch bedeutend für die Annahme des Anscheinsbeweis ist, dass bei der ec-Karte wegen der gesetzlich erforderlichen Überprüfung der Identität des Kunden bei Eröffnung des Kontos224 die Identität des Karten-Inhabers feststeht.

Ebenso zeigt die Diskussion um den Anscheinsbeweis, dass die materielle Rechtslage für die Frage, ob der Account-Inhaber selbst oder jemand mit seinem Einverständnis gehandelt hat, zunächst irrelevant ist. Erst wenn feststeht, dass dies nicht der Fall war, werden weitere Beweisthemen für die materielle Rechtslage, bei der ec-Karte die Frage der groben Fahrlässigkeit bei der Pflichtverletzung, relevant. Die Tatsache, dass bei der ec-Karte

218 Kollrus, MDR 2012, 377, 379; Schulte am Hülse/Welchering, NJW 2012, 1262, 1265.

$219 B G H$, Urteil v. 5. 10. 2004, XI ZR 210/03 - BGHZ 160, 308, 316.

220 Jungmann, in: Jahrbuch Junger Zivilrechtswissenschaftlicher 2007, 329, 345 f.; ders., ZZP 120 (2007), 459, 464.

221 Jungmann, ZZP 120 (2007), 459, 463 f. Dazu oben Rn. 788

222 Jungmann, in: Jahrbuch Junger Zivilrechtswissenschaftlicher 2007, 329, 347.

223 Ebd. 349.

224 Vgl. oben Rn.67 
vertragliche Beziehungen zwischen Karten-Inhaber und der Bank bestehen, was beim Missbrauch von Zugangsdaten im Internet häufig nicht der Fall ist, schadet daher einem Erkenntnisgewinn aus der Rechtslage bei der ecKarte nicht.

\section{Online-Banking}

819 Wegen der hohen praktischen Relevanz des Missbrauchs beim Online-Banking2 soll die Diskussion um Beweiserleichterungen der Bank für vermeintlich vom Kunden durchgeführte Transaktionen betrachtet werden, um Beweiserleichterungen für andere Zugangsdaten im Internet damit abgleichen zu können. Grundsätzlich kommt ein Anscheinsbeweis beim OnlineBanking nur in Betracht, wenn mit der herrschenden Meinung § 675w S. 3 BGB so verstanden wird, dass er die Rechtsfigur des Anscheinsbeweises nicht ausschließt ${ }^{226}$ Wegen der unterschiedlichen im Einsatz befindlichen Authentisierungsmethoden beim Online-Banking ist der Anscheinsbeweis differenziert zu betrachten.

820 Beim iTAN-Verfahren ${ }^{227}$ wird in Teilen der Literatur ein Anscheinsbeweis dafür angenommen, dass bei einer Transaktion mit den Zugangsdaten der Bankkunde selbst oder ein Vertreter mit Berechtigung behandelt hat 228 Dies ergebe sich aus der Übertragung des für die Verwendung der ec-Karte entwickelten Anscheinsbeweises ${ }^{229}$ weil das Online-Banking mit iTANVerfahren ein vergleichbaren Sicherheitsstandard besitze. Überwiegend wird der Anscheinsbeweis beim Online-Banking unter Verwendung des

225 Oben Rn. 67

226 Oben Rn. $\overline{812}$ sowie explizit zum Online-Banking Herresthal, in: Langenbucher/ Bliesener/Spindler, Kap. 5 \$675w BGB Rn. 13.

227 Dazu oben Rn. 556

228 Gössmann/Sönke, in: FS Nobbe, 93, 110; Hanau, Handeln unter fremder Nummer, S. 77; ders., VersR 2005, 1215, 1219 f.; Herresthal, in: Langenbucher/Bliesener/ Spindler, Kap. 5 § 675w BGB Rn. 14; Karper, DuD 2006, 215, 218; Recknagel, S. 149 ff.; S. Werner, MMR 1998, 232, 235; ders., in: Hoeren/Sieber/Holznagel, Kap. 13.5 Rn. 63; van Gelder, in: FS Nobbe, 55, 66 f.

229 Oben Rn. 812 
iTAN-Verfahrens abgelehnt ${ }^{230}$ Der Sicherheitsstandard reiche nicht aus. ${ }^{231}$ weil es sich beim iTAN-Verfahren um eine unsichere, rein wissensbasierte Authentisierungsmethode handelt 232 Die Möglichkeiten des Phishings und Pharming. ${ }^{233}$ Sowie von Man-in-the-Middle-Angriffer ${ }^{234}$ würden eine entsprechende Lebenserfahrung des Anscheinsbeweises widerlegen. Empirische Belege dafür, dass die Zugangsdaten häufig missbraucht werden, sprechen ferner gegen die Annahme eines Erfahrungssatzes, dass stets der Kontoinhaber oder ein Berechtigter gehandelt haben 235

Darüber hinaus sei die Bank am besten in der Lage, die Risiken eines Missbrauchs zu steuern, weswegen ein Anscheinsbeweis für unsichere Authentisierungsmethoden auszuschließen sei ${ }^{236}$ Dagegen wird eingewendet, dass die Bank keinen Einfluss auf die Konfiguration des Systems des Nutzers, wie Browser und Antivirensoftware nehmen könne 237 Ihr bleibt es jedoch unbenommen, Verfahren durchzusetzen, bei denen die Ausnutzung entsprechender Schwachstellen nicht möglich ist. Zusätzlich ist es dem Bankkunden faktisch schwer möglich einen Anscheinsbeweis zu erschüttern, weil er zur IT-Infrastruktur der Bank keine Angaben machen könne 238

Das mTAN- sowie das eTAN-Verfahren setzen hingegen auf eine ZweiFaktor-Authentisierung 239 Dadurch bieten sie einen besseren Schutz gegen Angriffe als eine rein wissensbasierte Authentisierung. Deswegen wird ein Anscheinsbeweis bei diesen Verfahren häufig angenommen ${ }^{240}$ Weil je-

230 LG Köln, Urteil v. 5. 12.2007, 9 S 195/07 - MMR 2008, 259, 261; AG Wiesloch, Urteil v. 20. 6. 2008, 4 C 57/08 - MMR 2008, 626, 628; Bergfelder, S. 283; Biallaß/Borges/Dienstbach u. a., in: Innovationsmotor IT-Sicherheit, 495, 507; Borges, NJW 2005, 3313, 3317; ders., BKR 2009, 85, 87; Casper, in: MüKoBGB $^{6}$ § 675w Rn. 20; Maihold, in: Schimansky/Bunte/Lwowski ${ }^{4}$, 55 Rn. 85; Mühlenbrock/Dienstbach, MMR 2008, 630; Omlor, in: Staudinger ${ }^{2012}$, § 675w BGB Rn. 10; Schulte am Hülse/Klabunde, MMR 2010, 84, 87; v. Westphalen, in: Erman $^{13}, \S 675$ w BGB Rn. 21.

231 Schulte am Hülse/Klabunde, MMR 2010, 84, 87.

232 Oben Rn. 545

233 Oben Rn. $\overline{138} \mathrm{ff}$.

234 Oben Rn. 168

235 Erfurth, WM 2006, 2198, 2205; Maihold, in: Schimansky/Bunte/Lwowski ${ }^{4}, \S 55$ Rn. 83; Recknagel, S. 142.

236 Erfurth, WM 2006, 2198, 2206.

237 Gössmann/Sönke, in: FS Nobbe, 93, 110.

238 Maihold, in: Schimansky/Bunte/Lwowski ${ }^{4}$, § 55 Rn. 81.

239 Dazu Oben Rn. 117 .

240 Casper, in: MüKo-BGB ${ }^{6}$ § 675w Rn. 20; Borges, BKR 2009, 85, 87; Mühlenbrock/ Dienstbach, MMR 2008, 630; Borges/Schwenk/Stuckenberg/Wegener, S. 313. 
doch auch Zwei-Faktor-Authentisierungsmethoden mit einem Man-in-theMiddle-Angriff angreifbar sind ${ }^{241}$ wird vereinzelt der Anscheinsbeweis dafür abgelehnt ${ }^{242}$ Nur das optimierte eTAN-Verfahren mit zwei komplett unabhängigen Komponenten reiche aus, um eine solche Beweiserleichterung anzunehmen 243 Anderes Stimmen der Literatur erkennen den Anscheinsbeweis nicht nur beim eTAN- sondern auch beim mTAN-Verfahren an 244 Wie das mTAN- und eTAN-Verfahren setzt das HBCI-Verfahren auch auf eine Zwei-Faktor-Authentisierung. Ein Anscheinsbeweis für das Handeln des Bankkunden solle daher bei diesem Verfahren bestehen 245 Dies wird insbesondere mit der Nähe zur elektronischen Signatur begründet ${ }^{246}$ Teilweise wird jedoch ein Anscheinsbeweis beim HBCI-Verfahren abgelehnt ${ }^{247}$ weil sich die Definition des Sicherheitsstandards zu weit von der elektronischen Signatur bewegt habe.

823 Wird der Anscheinsbeweis beim Online-Banking abgelehnt oder schafft es der Bankkunde einen solchen Anscheinsbeweis zu erschüttern, kommt eine Haftung wegen grob fahrlässigen Umgangs mit den Zugangsdaten nach $\S 675 v$ Abs. 2 BGB in Betracht. Dabei kann ebenfalls ein Anscheinsbeweis für die Pflichtverletzung des Bankkunden erwogen werden. Im Gegensatz zur Rechtslage bei der ec-Karte ${ }^{248}$ wird dieser Anscheinsbeweis für die Pflichtverletzung beim Online-Banking überwiegend abgelehnt ${ }^{249}$

824 Eine Beweiserleichterung in Form des Anscheinsbeweises steht der Bank somit bei einer rein wissensbasierten Authentisierung nach einer überwiegenden Ansicht nicht zur Verfügung. Eine Beweiserleichterung der Bank in Form einer sekundären Darlegungslast solle ihr jedoch für solche Tatsachen zu Gute kommen, die dem Bankkunden, jedoch nicht ihr, bekannt sind 250

\section{Oben Rn. 146}

242 Maihold, in: Schimansky/Bunte/Lwowski ${ }^{4}$, § 55 Rn. 86.

243 Ebd., § 55 Rn. 86.

244 Herresthal, in: Langenbucher/Bliesener/Spindler, Kap. 5 § 675w BGB Rn. 15.

245 Gössmann/Sönke, in: FS Nobbe, 93, 110; Omlor, in: Staudinger ${ }^{2012}$, § 675w BGB Rn. 10; Recknagel, S. 150.

246 Gössmann/Sönke, in: FS Nobbe, 93, 113; Recknagel, S. 150.

247 Maihold, in: Schimansky/Bunte/Lwowski ${ }^{4}$, § 55 Rn. 84; Bergfelder, S. 287; v. Westphalen, in: Erman ${ }^{13}, \S 675$ w BGB Rn. 21.

248 Oben Rn. 813

249 KG Berlin, Urteil v. 29. 11. 2010, 26 U 159/09 - MMR 2011, 338; LG Mannheim, Urteil v. 16. 5. 2008, 1 S 189/07 - MMR 2008, 765; Maihold, in: Schimansky/Buntel Lwowski $^{4}$, § 55 Rn. 166 ff.; a.A. Gössmann/Sönke, in: FS Nobbe, 93, 110.

250 Maihold, in: Schimansky/Bunte/Lwowski ${ }^{4}$, § 55 Rn. 88. 
Der differenzierten Diskussion um den Anscheinsbeweis beim OnlineBanking lassen sich Wertungen entnehmen, die für Beweiserleichterungen bei anderen Zugangsdaten im Internet relevant werden können. Die rein wissensbasierte Authentisierung bietet keine ausreichende Grundlage für die Anerkennung eines Anscheinsbeweises. Bei einer Zwei-Faktor-Authentisierung kann ein solcher jedoch durchaus angenommen werden. Wegen verbleibender Angriffsmöglichkeiten wird jedoch an der Anerkennung des Anscheinsbeweises gezweifelt. Dabei wird stets ein Vergleich zu der Situation des $\S 371$ a Abs. 1 S. 2 ZPO bemüht und die Vergleichbarkeit mit dem Sicherheitsstandard der qualifizierten elektronischen Signatur angestellt. Wie bei der ec-Karte ist für den Anscheinsbeweis beim Online-Banking zwar unausgesprochen aber entscheidend, dass die Identität des Bankkunden bei der Eröffnung des Konto zuverlässig überprüft wird ${ }^{251}$ Die Zuordnung des Kontos zu seinem Inhaber steht damit ausreichend sicher fest. Ebenso wie bei der ec-Karte ${ }^{252}$ beschäftigt sich der Anscheinsbeweis nur mit der tatsächlichen Frage, ob der Bankkunde oder ein Dritter mit seinem Einverständnis gehandelt hat, sodass die vertraglichen Beziehungen, die bei anderen $\mathrm{Zu}$ gangsdaten im Internet häufig nicht vorliegen, für die Beweiserleichterung keine Rolle spielen.

\section{Zwischenergebnis}

Aus der Betrachtung verschiedener anerkannter Beweiserleichterungen bei ähnlichen Konstellationen lassen sich einige Wertungen herausfiltern, die für Beweiserleichterungen beim Missbrauch von Zugangsdaten im Internet relevant werden. Zunächst dreht sich ein Großteil der Diskussionen um die Frage, ob ein Anscheinsbeweis in Betracht kommt. Andere Formen der Beweiserleichterungen werden kaum diskutiert. Über die Gründe, warum stärkere Beweiserleichterungen wie die tatsächliche Vermutung außer Acht gelassen werden, kann nur spekuliert werden. Vermutlich erfolgt die Abgrenzung von Anscheinsbeweis und tatsächlicher Vermutung wegen der ähnlichen Voraussetzungen 253 insbesondere anhand der Rechtsfolge. Trifft dies zu, kann aus den Diskussionen der soeben behandelten Fälle geschlossen 
werden, dass die Rechtsfolge der Beweislastumkehr bei den ähnlichen Konstellationen für unangemessen erachtet wird.

827 Für den Anscheinsbeweis lassen die soeben untersuchten Konstellationen einige Schlüsse zu. Eine rein wissensbasierte Authentisierung reicht für die Anerkennung des Anscheinsbeweises wegen der zahlreichen Missbrauchsmöglichkeiten nicht aus. Eine Zwei-Faktor-Authentisierung hingegen kann wegen der erhöhten Sicherheit, insbesondere wegen der Kombination aus der physischen Einmaligkeit der Besitz-Komponente und der Geheimhaltung des Wissens-Komponente, eine ausreichende Grundlage sein. Diese Wertung deckt sich mit den Erkenntnissen zum Rechtsscheintatbestand 254 Ebenso hat die Analyse der verschiedenen Beweiserleichterungen gezeigt, dass die Identifizierung des Account-Inhabers eine wichtige Rolle spielt. Dies deckt sich ebenfalls mit den Erkenntnissen des Rechtsscheintatbestandes 255

828 Ferner lässt sich aus den untersuchten Konstellationen die unausgesprochene Tatsache herausfiltern, dass die Erklärung im Nachhinein nicht verändert wurde. Die elektronische Signatur dient primär dazu, dass überprüft werden kann, dass ein Text unverändert ist. Bei der ec-Karte bieten das Journal des Geldautomaten sowie die Autorisierungsprotokolle 256 ausreichende Gewähr dafür, dass die Buchungsvorgänge nicht nachträglich verändert wurden. Für die Anerkennung eines Erfahrungssatzes, der einen Anscheinsbeweis begründen kann, ist daher ebenso wie beim Rechtsscheintatbestand erforderlich, dass eine ausreichend sichere Authentisierungsmethode verwendet wird, die die virtuelle Identität des Accounts durch eine Identitätsüberprüfung zuverlässig einer numerischen Identität zuordnet 257 Darüber hinaus muss eine nachträgliche Fälschung der abgegebenen Erklärung unwahrscheinlich sein.

829 Aus den betrachteten Konstellationen lässt sich daher die folgende Schlussfolgerung ziehen: Wenn eine ausreichend sichere Authentisierungsmethode wie die Zwei-Faktor-Authentisierung verwendet wurde, die virtuelle Identität des Accounts zuverlässig der numerischen Identität des Account-Inhabers zugeordnet ist und eine nachträgliche Fälschung der Er-

254 Oben Rn. $534 \mathrm{ff}$.

255 Oben Rn. $\overline{595} \mathrm{ff}$.

256 Dazu Maihold, in: Schimansky/Bunte/Lwowski ${ }^{4}$, § 54 Rn. 10, 115.

257 Ohne das Erfordernis der Identitätsprüfung auch Borges, Elektronischer Identitätsnachweis, S. 230. Nur die Authentisierungsmethode sei entscheidend Kuhn, S. 254. 
klärung unwahrscheinlich ist, besteht ein Anscheinsbeweis dafür, dass der Account-Inhaber die in Frage stehende Erklärung über den Account selbst abgegeben hat. Sind diese hohen Anforderungen für den Anscheinsbeweis bei der Art des Accounts nicht gegeben, kann eine Beweiserleichterung in Form der sekundären Darlegungslast nach deren allgemeinen Voraussetzungen in Betracht kommen. 
